\title{
Surveying the global landscape of post-transcriptional regulators
}

\author{
Kendra Reynaud', Anna McGeachy², David Noble2, Zuriah Meacham², Nicholas Ingolia1,2* \\ 1Biophysics Graduate Group, California Institute for Quantitative Biosciences, and 2Department of Molecular and Cell \\ Biology, University of California, Berkeley, Berkeley, California, United States of America \\ * Correspondence: ingolia@berkeley.edu
}

Numerous proteins regulate gene expression by modulating mRNA translation and decay. In order to uncover the full scope of these post-transcriptional regulators, we conducted an unbiased survey that quantifies regulatory activity across the budding yeast proteome and delineates the protein domains responsible for these effects. Our approach couples a tethered function assay with quantitative single-cell fluorescence measurements to analyze $\sim 50,000$ protein fragments and determine their effects on a tethered mRNA. We characterize hundreds of strong regulators, which are enriched for canonical and unconventional mRNA-binding proteins. Regulatory activity typically maps outside the RNA-binding domains themselves, highlighting a modular architecture that separates mRNA targeting from post-transcriptional regulation. Activity often aligns with intrinsically disordered regions that can interact with other proteins, even in core mRNA translation and degradation factors. Our results thus reveal networks of interacting proteins that control mRNA fate and illuminate the molecular basis for post-transcriptional gene regulation.

A dynamic network of proteins regulates the expression of messenger RNA (mRNA) to maintain homeostasis and adapt cell physiology to changing environments ${ }^{1}$. This network entails a complex interplay between cis-acting sequence element encoded in the mRNA and trans-acting factors that bind the transcript to regulate its fate 2. RNA-binding proteins (RBPs) determine whether a given mRNA is translationally activated or repressed, localized to a specific region or compartment within the cell, or degraded ${ }^{1}$. In addition, RBPs can remodel RNA structure to make it more accessible to other RBPs or enzymes and can act as chaperones to prevent RNA aggregation and misfolding 3,4. Thus, determining which RBPs mediate distinct regulatory programs is critical to understanding post-transcriptional control of gene expression.

Efforts to understand the diversity of RBPs and identify their mRNA targets have revealed general principles of protein-RNA interactions. Several recurring RNA binding domains (RBDs) can individually recognize 4-9 nucleotide motifs in RNA and often appear in various combinations to achieve greater specificity ${ }^{5-7}$. Furthermore, protein-RNA crosslinking reveals a diverse mRNA interactome that includes many proteins without canonical RBDs 8 , including approximately 700 high-confidence RNA-protein interactions in budding yeast 8,9 . Reciprocally, crosslinking and immunoprecipitation (CLIP) experiments have defined the mRNA targets for hundreds of these RNA-binding proteins 2,10,11. Taken together, these approaches expose a dense web of interactions suggesting complex patterns of post-transcriptional regulation.

Though these high-throughput experiments have revealed principles and targets of RNA binding, we lack an understanding of the functional impact of these proteins on their target mRNAs. Traditional approaches rely on measuring how individual RBPs regulate their direct mRNA targets 12 and examining how these targets change when the protein is perturbed ${ }^{13}$. These approaches are valuable, but they do not provide a scalable approach to characterize the regulatory networks underlying posttranscriptional regulation.

The modular architecture of regulatory RBPs 5 spurred the development of the tethered function assay to bypass the endogenous RNA 
specificity of RBPs and instead recruit them to a heterologous reporter ${ }^{14}$. This approach can interrogate the regulatory effects of proteins or isolated domains, and it can reveal the function of regulatory cofactors that do not themselves bind RNA directly ${ }^{15}$. In the tethering assay, candidate regulatory proteins are targeted to a reporter transcript using heterologous, bacteriophage-derived protein-RNA interaction pairs. These targeting systems utilize a specific, high-affinity interaction between a bacteriophage coat protein and a cognate RNA hairpin to recruit potential regulators to the reporter, obviating whatever direct RNA binding or indirect interactions might recruit a protein to its endogenous targets. This independence from endogenous target mRNAs and compatibility with robust reporters such as luciferase makes the tethering assay well-suited to highthroughput characterization of posttranscriptional regulators. Indeed, tethering assays have revealed over 50 regulatory proteins in a systematic analysis of 700 full-length human RBPs ${ }^{16}$. It is also amenable to unbiased screening, as demonstrated by identification of almost 300 post-transcriptional regulators in trypanosomes ${ }^{17}$. These studies highlight the value of tethered function assays for systematic and comprehensive study of post-transcriptional regulation.

In this study, we adapt the tethering assay to survey regulatory activity across the entire yeast proteome in an unbiased manner. Our approach allowed us to identify hundreds of proteins that modulate mRNA translation and stability, including highly active, non-canonical RBPs. We subdivided proteins and mapped their regulatory activity to particular domains and regions, in some cases uncovering effects that are not apparent in the context of the full-length protein. This fine resolution allowed us to identify Pfam protein domains and short peptide motifs that were enriched amongst the most active post-transcriptional regulators. Notably, although canonical RBPs were strongly enriched among the active regulators, the regulatory activity generally mapped outside the RBD. Taken together, our work provides a systematic, functional characterization of posttranscriptional regulators in budding yeast, thereby expanding our understanding of the complex network of proteins that control RNA metabolism.

\section{RESULTS}

\section{Functional characterization of post- transcriptional regulators with a quantitative fluorescence tethering assay}

We set out to functionally assess the RNA regulatory activity of proteins across the entire yeast proteome through the tethered-function assay. These tethering assays have typically used luciferase reporters 15 , which have been adapted to measure hundreds of RBP tethering constructs in an arrayed format ${ }^{16}$. In order to perform an unbiased survey of the yeast proteome not restricted to annotated RBPs, we needed to analyze libraries on a substantially larger scale.

Flow cytometry provides high-throughput, single-cell phenotypic measurements and enables large, pooled screens using fluorescenceactivated cell sorting (FACS). FACS analysis of tethered function assays relies on fluorescent protein reporters 17 , and so we devised a budding yeast tethering assay coupled to a ratiometric fluorescence readout. We target a transcript encoding a yellow fluorescent protein (YFP) by tethering it to a candidate regulatory protein, which will change YFP expression and thus yellow fluorescence. Our fluorescent reporter transcripts contained five boxB RNA hairpins in their $3^{\prime}$ UTR, which are recognized by the $\lambda \mathrm{N}$ coat protein ${ }^{18}$. In order to control for non-specific changes in cell size and physiology, we normalize these yellow fluorescence measurements against red fluorescence expressed from a control red fluorescent protein (RFP) transcript that is not targeted by $\lambda \mathrm{N}$. Changes in the ratio of fluorescence intensity between the yellow reporter and the red control 
a

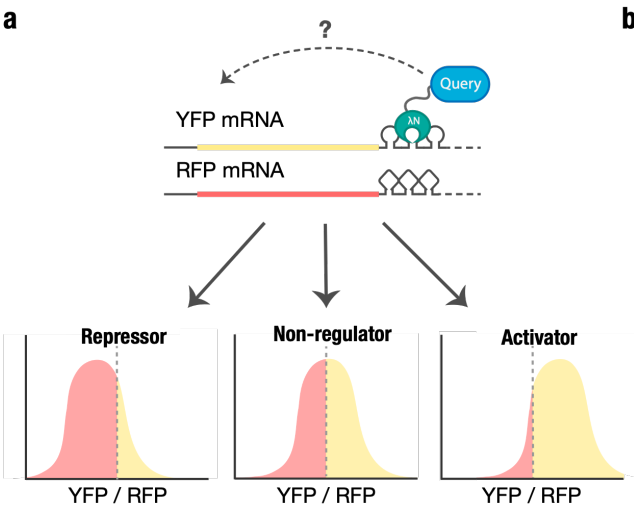

C
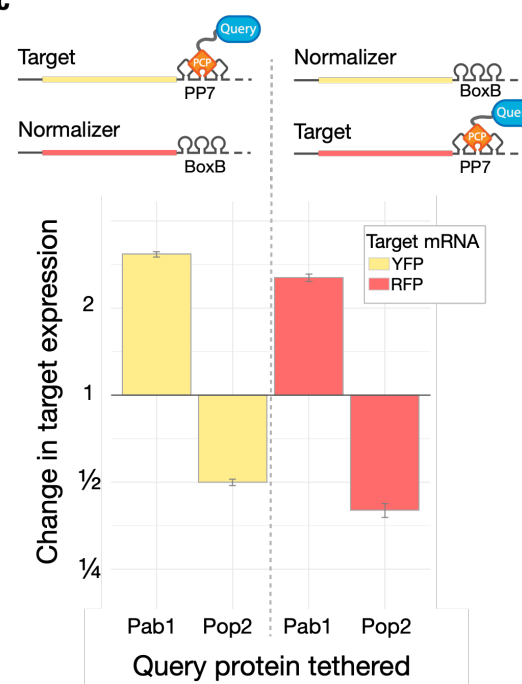

provide a high-precision, quantitative measurement of specific regulatory activity affecting the targeted mRNA while controlling for global effects that also affect the non-targeted mRNA (Fig. 1a). To further control for the possibility that binding of $\lambda \mathrm{N}$ itself affects the reporter, we normalize the fluorescence ratio of tethered fusion constructs against a tethered Halo protein, which exhibits no inherent regulatory effect.

We validated our assay by measuring how wellcharacterized regulators affected reporter expression. Tethered poly(A)-binding protein (Pab1 in budding yeast) is known to enhance reporter expression by stabilizing mRNA ${ }^{14}$ and promoting its translation ${ }^{19}$. Conversely, the CCR4-NOT complex is responsible for the majority of cytosolic mRNA deadenylation ${ }^{20}$, and tethering of the CAF1 deadenylase (Pop2 in budding yeast) greatly destabilizes target mRNAs ${ }^{21}$. We observed $\sim 3$-fold target RNA activation by tethered Pab1- $\lambda \mathrm{N}$, and $\sim 5$-fold reporter repression by tethered Pop $2-\lambda \mathrm{N}$, relative to an inactive HaloTag- $\lambda \mathrm{N}$ control (Fig. $1 b)$. We observed quantitatively consistent effects when we switched our tethering assay to target the RFP transcript and normalized against a nontargeted YPF control, ensuring that the regulatory effects we measured were independent of our choice of fluorescent reporter protein (Fig. 1b). We further tested that they did not depend on the particular choice of the $\lambda \mathrm{N} \bullet$ boxB interaction pair by tethering Pab1 and Pop2 to reporters containing one PP7 hairpin using fusions with the PP7 coat protein (PP7cp) 18. Both PP7cp fusions showed similar activity on their cognate targets as $\lambda \mathrm{N}$, although Pop2PP7cp repression appeared weaker than Pop2$\lambda \mathrm{N}$ repression, potentially due to the use of only 
a single PP7 hairpin (Fig. 1c). We went on to measure the activity of the RNA-binding protein Sgn1, a poorly-characterized protein that seems to be involved in regulation of translation based on co-immunoprecipitation with Pab1 and negative genetic interactions with mutations in the Pab1-interacting translation initiation factor eIF4G (Extended Data Fig. 1b) 22. We found that Sgn1 served as a powerful activator that upregulated YFP expression by over 6-fold relative to RFP (Fig. 1d), in addition to modestly increasing RFP levels and cell size (Extended Data Fig. 1c-f). Sgn1 tethering increased target RNA abundance roughly 2.5-fold (Extended Data Fig. 1g), and based on the larger change we see in YFP fluorescence, we infer that it activates translation as well. Taken together, these results confirm that our tethering assay provides robust and quantitative measurements of mRNAspecific regulatory activity, even in the face of additional non-specific effects on the cell, and thus provides a powerful tool for a highthroughput, proteome-wide survey of mRNA regulators.

\section{An unbiased survey of the yeast proteome identifies hundreds of active post-transcriptional regulators}

We set out to survey the yeast proteome comprehensively for post-transcriptional regulatory activity using our two-color fluorescent tethering assay in conjunction with cell sorting and sequencing. We sought first to create a large library of $\lambda \mathrm{N}$ fusion constructs and introduce this library into yeast in order to establish a pool of cells that each expressed one tethering fusion protein. FACS then allowed us to sort these cells into sub-populations according to their fluorescence phenotypes. Deep sequencing quantified the tethering constructs in each of these sorted groups. Tethering protein fusions with regulatory activity would alter the fluorescence phenotype of the host cell, shifting it into a subpopulation with an unusually low or high fluorescence ratio (Fig. 1a), and altering the distribution of the active tethering fusion across the sorted cells.

We began by generating a proteome-scale library of $\lambda \mathrm{N}$ fusions that would enable unbiased discovery of regulatory proteins and identification of functional domains within these regulators. We reasoned that we could construct an unbiased $\lambda \mathrm{N}$ fusion library directly from randomly fragmented genomic DNA, as budding yeast has a compact and intron-poor genome. Such a library should provide uniform representation of all proteins encoded in the proteome, based on their equal DNA copy number. Only a fraction of random genomic DNA fragments will capture the correct strand and frame of a gene, however, and so we required an additional selection for in-frame fragments. We first generated fragments by transposon-mediated tagmentation 23,24 and selected fragments of roughly 500 base pairs in order to capture whole protein domains, which have a typical size of $\sim 100$ amino acids 25 (Fig. 2a and Extended Data Fig. 2a). We then captured these fragments by gap repair into a vector that depended on in-frame translation through the intervening sequence in order to express a downstream selectable marker (Fig. 2b). After selection, we analyzed ten individual clones and found that all ten encoded in-frame fusions, indicating that we successfully enriched for productive fusions (Extended Dataset 1). We then transferred our fragment library into a $\lambda \mathrm{N}$ fusion expression vector and added random, 25nucleotide barcodes that identify each fragment uniquely (Extended Dataset 2) 26,27. The mean fragment size in our barcoded $\lambda \mathrm{N}$ fusion library was about 500 base pairs, consistent with the fragment size of the genomic DNA input (Fig. 2c), and we captured at least one representative fragment from roughly half of all yeast genes.

We then analyzed the regulatory activity of each individual protein fragment in our library by pooled transformation, flow sorting, and sequencing. We transformed the $\lambda \mathrm{N}$ fusion library into a yeast strain expressing our dual 
bioRxiv preprint doi: https://doi.org/10.1101/2021.08.09.455688; this version posted August 9, 2021. The copyright holder for this preprint (which was not certified by peer review) is the author/funder, who has granted bioRxiv a license to display the preprint in perpetuity. It is made available under aCC-BY-NC 4.0 International license.

Reynaud et al.

Surveying Post-transcriptional Regulators

a
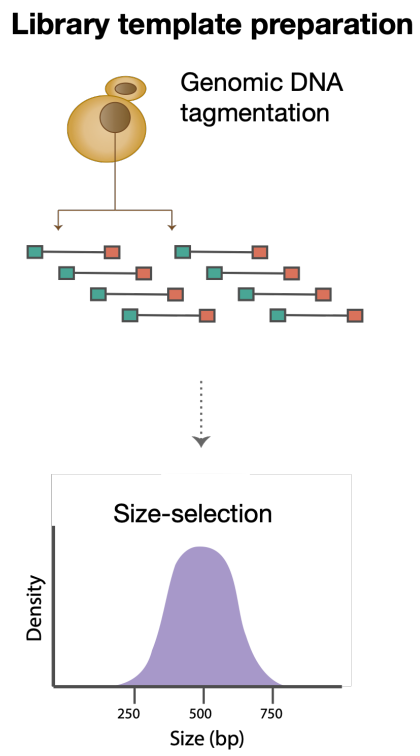

b

In-frame fragment selection
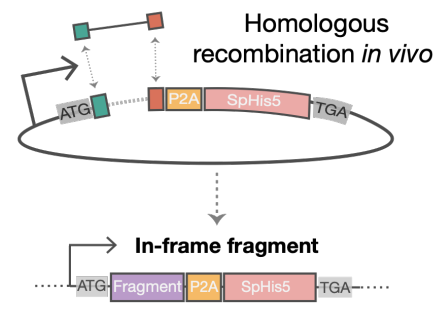

Proteins encoded:

Growth in

selective media:

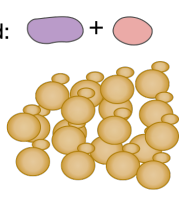

$\longrightarrow$ Out-of-frame fragment

Protein truncated:

No growth in

selective media:
C

\section{Library transfer and barcoding}

Library subcloned Fragments barcoded
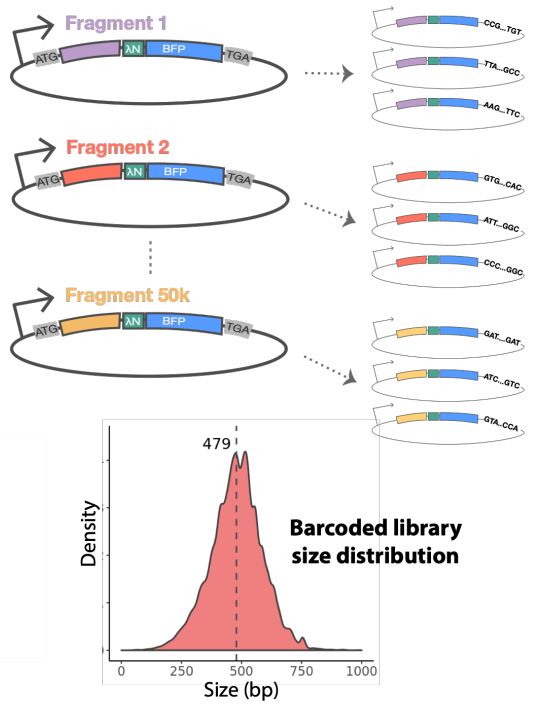

Sort cells by fluorescence ratio
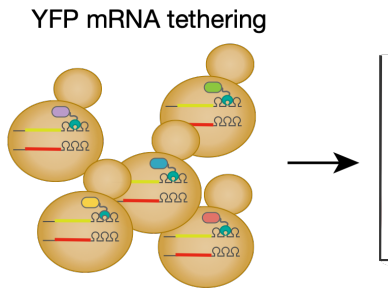

Isolate plasmid DNA
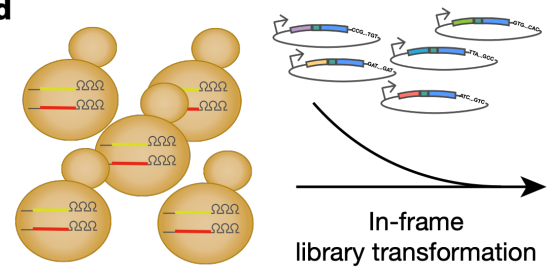

library transformation

e

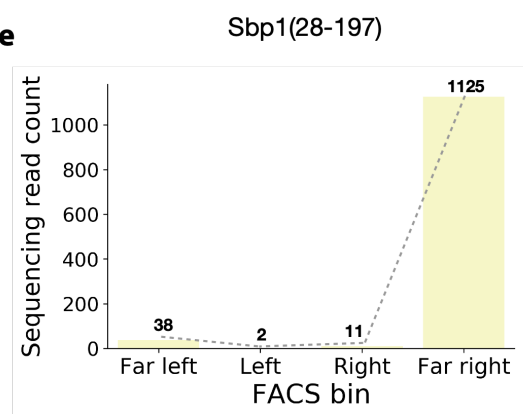

f

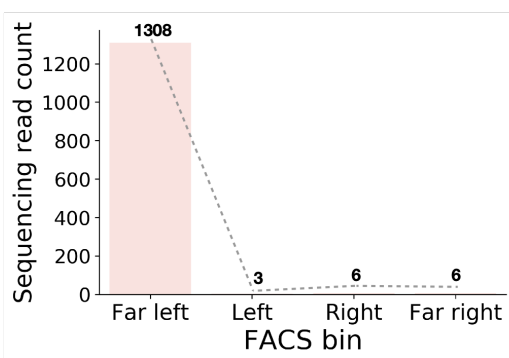

Ebs1(708-876)

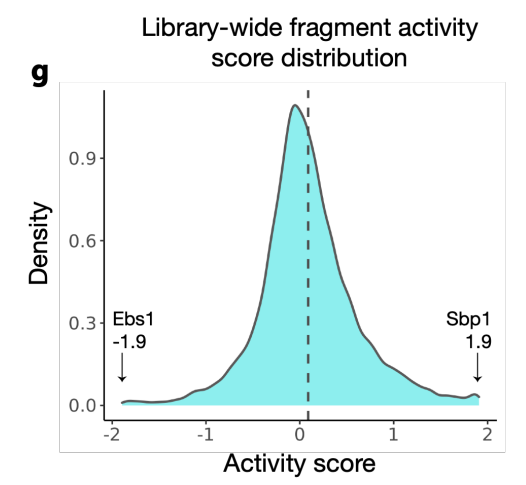

Fig. 2: Generating an unbiased, proteome-wide survey of tethered in-frame protein fragments.

a, S288C genomic DNA was fragmented by transposon-mediated tagmentation and selected to recover fragments with an average size of 500 base pairs. b. DNA fragments were cloned by in vivo gap repair into a plasmid containing a downstream selectable marker. Fragments containing an open reading frame in the correct phase will express a functional S. pombe HIS5 gene and support growth on selective media, whereas cells harboring out-of-frame fragments will fail to grow. c, Selected fragments were subcloned into the tethering vector with the $\lambda \mathrm{N}$ and BFP proteins encoded downstream. Fragments were assigned on average three barcodes each. The barcoded library size distribution did not significantly change from in the initial input fragment library. $\mathbf{d}$, The library was transformed into the dual-reporter yeast strain where the fragments were tethered to YFP mRNA. Phenotypic changes were captured by FACS sorting based on YFP versus RFP expression. Plasmids encoding the tethered fragments were isolated, and the barcodes associated with each fragment were amplified and then quantified through next-generation sequencing. e, Distribution of read counts per FACS bin for Sbp1(28-197) activator fragment. f, As in e, for Ebs1(708-876) repressor fragment. g, Kernel density estimate (KDE) of the library-wide activity score distribution. 
fluorescent protein reporters and sorted these transformed cells into four sub-populations of equal size according to YFP/RFP fluorescence ratio. We then isolated library plasmid DNA from our sorted cells and quantified the barcodes by next-generation sequencing (Fig. $2 \mathrm{~d}$ ). We expected activators to be enriched in bins with higher YFP / RFP ratios, while repressors should be enriched in bins with lower ratios.

Indeed, we saw certain tethering constructs that displayed a dramatic skew in their abundance across the sorted cells. For example, one particular fragment of the RNA-binding protein Sbp1 was sorted almost entirely into the highest YFP gate, indicating it strongly activated reporter expression (Fig. 2e). Conversely, a fragment of the nonsense-mediated decay factor Ebs1 acted as a strong repressor that was found almost exclusively in the lowest YFP subpopulation (Fig. 2f). To quantify this enrichment, we derived an "activity score" for each fragment in our screen by computing a maximum likelihood estimate of its average fluorescence value, expressed as a $z$-score relative to the overall population. In our sorting scheme, these scores ranged from -1.9 for strong repressors like Ebs1 to +1.9 for strong activators like Sbp1. As anticipated, most fragments in our library had activity scores close to zero, indicating little or no effect on reporter transcript expression (Fig. $2 \mathrm{~g}$ and Extended Dataset 3). Pooled screening identified active fragments from many wellknown regulatory proteins such as the DEADbox RNA helicase Ded1, a translation initiation factor $28-30$, and Ngr1, which induces the decay of POR1 mRNA ${ }^{31}$. Our unbiased approach also uncovered novel post-transcriptional regulation in proteins with other well-characterized cellular functions, including the small heat shock chaperone Hsp26, which also has previouslyidentified mRNA binding activity ${ }^{32}$.

Furthermore, we uncovered regulatory regions in proteins of unknown function, like Her1, which may interact with ribosomes based on copurification experiments ${ }^{33}$. These results illustrate the power of our approach to discover proteins that control mRNA stability and translational efficiency and quantify how this affects gene expression.

\section{Full-length proteins display qualitatively similar regulatory activity as truncated fragments} We selected twelve fragments across a range of activity scores and biological functions (Fig. 3a) and tested them individually in the tethering assay by direct fluorescence measurements. All twelve fragments shifted the fluorescence ratio in the direction matching their effect in the largescale survey (Fig. 3b), and we found a strong correlation between the magnitude of the change in YFP expression in the tethering assay and the activity score in the screen $(r=0.91)$ (Fig. 3c). This strong quantitative agreement demonstrates that the activity score derived from sorting and sequencing is an accurate measure of the regulatory effect of a fragment and can be used to discover proteins involved in posttranscriptional regulation.

Isolated protein fragments may have different activities than the full protein from which they are derived due to the absence of regulatory domains, altered protein-protein interactions, or changes in localization, among others. We thus selected a handful of active fragments to explore how their activity relates to the wild-type protein. Full-length Sbp1 is an RBP with two RRMs in addition to an RGG-motif in the middle of the protein that recruits Pab1 ${ }^{34}$. The fragment that we characterized as a $\sim 3$-fold activator (Fig. $2 \mathrm{e})$ contained only the first RRM and the RGG motif, whereas the full-length version of the protein was a weaker, $\sim 2$-fold activator (Fig. 3d). We hypothesize that the inclusion of the second RRM interferes with its ability to recruit Pab1 as efficiently to the reporter $3^{\prime}$ UTR, making it a weaker activator. In other cases, the full-length protein had a stronger effect than the isolated fragment. Sro9 is an RBP that contains a La-motif and is hypothesized to activate translation through recruitment of the closed-loop-forming translation initiation complex ${ }^{35}$. We identified an 
bioRxiv preprint doi: https://doi.org/10.1101/2021.08.09.455688; this version posted August 9, 2021. The copyright holder for this preprint (which was not certified by peer review) is the author/funder, who has granted bioRxiv a license to display the preprint in perpetuity. It is made available under aCC-BY-NC 4.0 International license.

Reynaud et al.

a

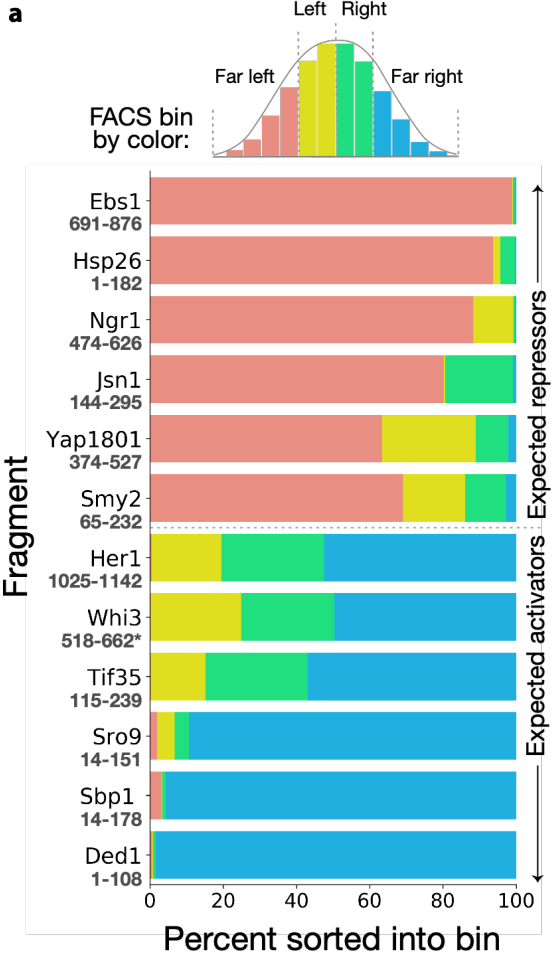

c

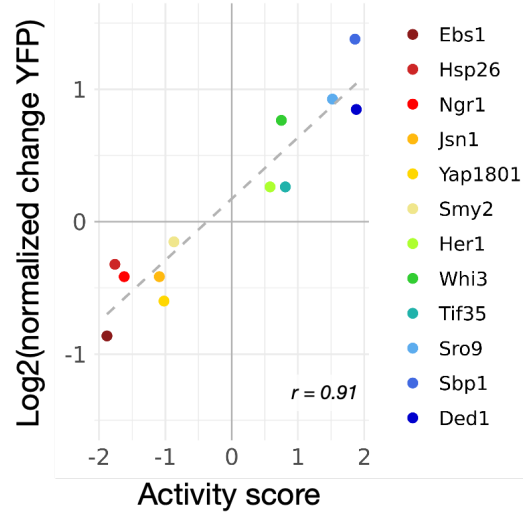

Surveying Post-transcriptional Regulators b
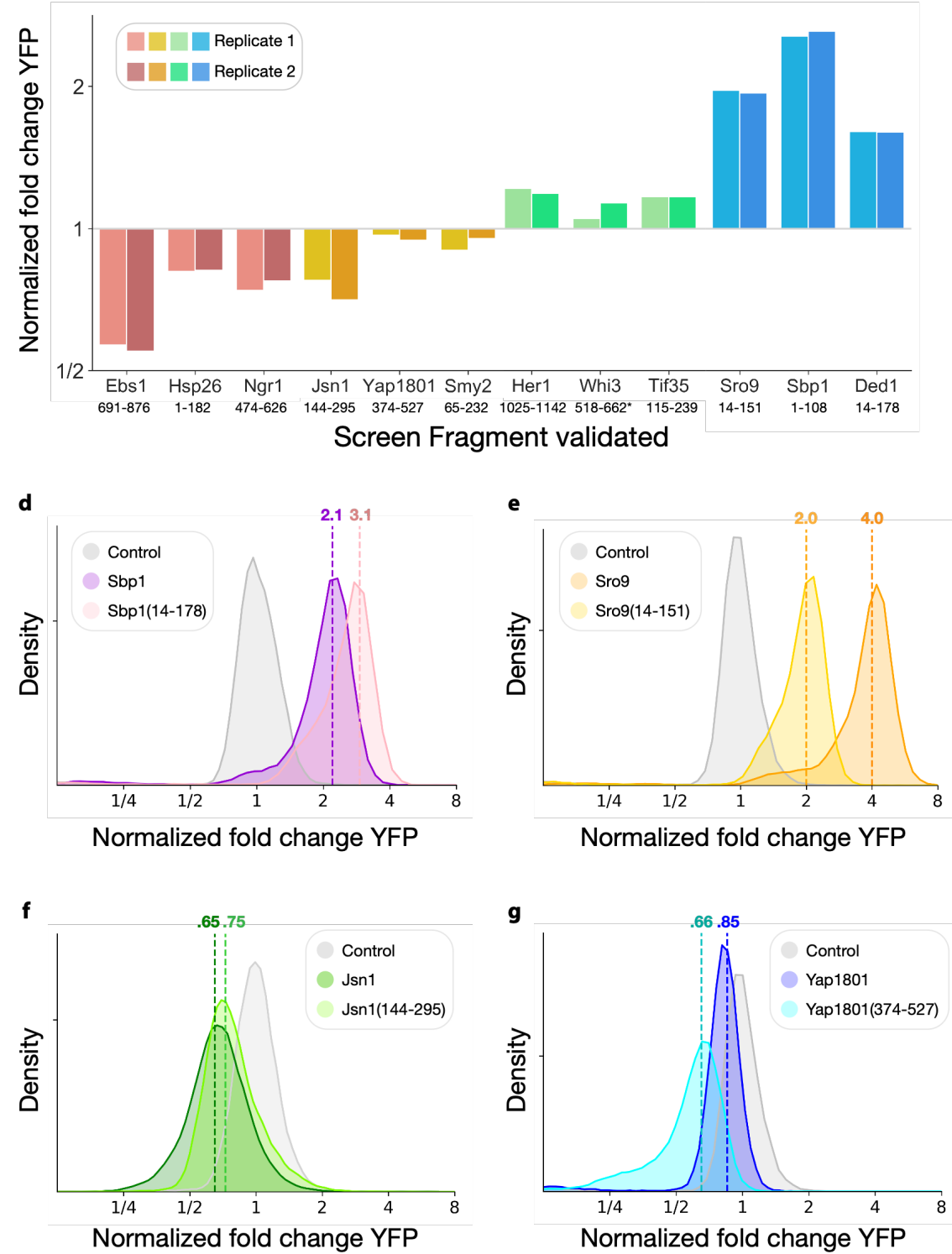

Fig. 3: Protein fragment activity in the tethering screen represents real, verifiable regulatory function.

a, Distribution of sequencing reads across sub-populations separated by FACS. $\mathbf{b}$, Median activity of each protein fragment in the flow cytometry tethering assay $\left(n=2\right.$ per fragment). $c$, Comparison of the $\log _{2}$ (difference in fluorescence ratio) and the screen activity score per fragment, $r=0.91$. d, Flow cytometry measuring activity of Sbp1 and Sbp1(14-178) in the tethering assay $(\mathrm{n}=2$, one replicate per sample shown). e, As in $\mathbf{d}$, for Sro9 and Sro9(14-151) ( $\mathrm{n}=2$, one replicate per sample shown). $\mathbf{f}$, As in $\mathbf{d}$, for Jsn1 and Jsn1(144-295) ( $\mathrm{n}=2$, one replicate per sample shown). $\mathbf{g}$, As in $\mathbf{d}$, for Yap1801 and Yap1801(374-527) ( $\mathrm{n}=$ 2, one replicate per sample shown).

Sro9 fragment that activated expression $\sim 2$-fold, whereas the full-length protein was an even more robust activator and increased reporter expression by nearly 4-fold (Fig. 3e). Tethering the entire yeast Puf-domain protein Jsn1 likewise produced a stronger repressive effect than the fragment we identified in our tethering library (Fig. 3f). In contrast, the intact version of the endocytic protein Yap1801 ${ }^{36}$ was less repressive than our fragment (Fig. 3g). One possible explanation for this effect may be that Yap1801(374-527) is involved in localization of the protein to the plasma membrane where the mRNA is less actively-translated ${ }^{37}$. Nonetheless, in all four cases, the full-length protein exerted an effect in the same direction as the fragment 
tested in our screen. Our approach is thus well suited to survey the regulatory activity contained in the native proteome and ascribe functions to RNA-binding proteins.

\section{Active regulators are enriched in RNA-binding proteins but not in RNA-binding domains}

Our tethering assay can detect regulatory activity in truncated proteins lacking RNAbinding domains and in proteins that lack any intrinsic RNA-binding activity. This feature of the assay allows us to study the regulatory activity of co-regulators that may not bind RNA directly. Nonetheless, we did expect a substantial overlap between the post-transcriptional regulators detected in the screen and previouslyknown RBPs. In order to test this hypothesis, we compiled a list of budding yeast RBPs from proteins appearing in at least two of four overlapping datasets that reported RNA-protein interactions (Fig. 4a) ${ }^{38-41}$. We found that fragments from these known RPBs had substantially higher absolute activity scores than the overall proteome, indicating that RBPs were more likely to show activity in our screen (Fig. $4 b)$. This association between RBPs and regulatory activity further confirmed the relevance of our results for endogenous programs of post-transcriptional regulation controlled by these RBPs. It also raised the question whether regulatory activity was associated with the RNA-binding domains of these RBPs or with other regions of the protein.

Our tethering screen measured the activity of truncated protein fragments, and so we were able to ascribe quantitative regulatory effects to particular regions and domains of the overall protein. We were thus able to investigate which protein domains were enriched amongst the most active fragments in our screen, and in particular, whether these active regions coincided with RNA-binding domains. For each fragment, we first determined whether it contained a protein domain from the Pfam database ${ }^{42}$. The boundaries of our random fragments did not align perfectly with protein domain boundaries, and so we restricted our domain enrichment analysis to library fragments containing at least $75 \%$ of some Pfam protein family. We then tested each family individually to determine whether the activity scores of fragments from that family were significantly higher or lower than the library overall (Fig. 4c).

Dozens of protein families were associated with active regulators in this analysis, and some of the strongest associations involved domains with clear connections to translation and RNA decay (Extended Dataset 6). We observed the strongest positive mean activity score amongst fragments derived from the translation initiation factor eIF3 43. We also saw a trend for activators among the DEAD box helicase family proteins, which include translation initiation factors eIF4A and Ded1 ${ }^{44}$. The endo/exonuclease/ phosphatase family showed up among the strong repressors; these include certain subunits of the Ccr4-Not complex, for example ${ }^{42}$. Other domains, such as Vacuole-related protein 17, which moves the vacuole along actin cables into the bud ${ }^{45}$, may act through mRNA localization. We also saw many families encoding metabolic functions such as adenylosuccinate synthetase 46, FADdependent oxidoreductase, and malic enzyme Nterminal domain. Metabolic enzymes have emerged as cryptic RNA-binding proteins ${ }^{9}$, and so it seems noteworthy that they appear to show regulatory activity as well. Notably, although many canonical RNA-binding domains such as RRMs appear in Pfam, they were not enriched in the active fragments. The absence of canonical RNA-recognition domains from the enriched Pfam domains indicates that RBDs are more important for mRNA target selection, and that the regions adjacent to the RNA-interacting domains typically provide regulatory activity for the RBP.

Our screen identified strong activity in fragments lacking an identifiable, folded domain. Indeed, many proteins contain intrinsically-disordered regions (IDRs) in addition to folded domains. Such IDRs play 
bioRxiv preprint doi: https://doi.org/10.1101/2021.08.09.455688; this version posted August 9, 2021. The copyright holder for this preprint (which was not certified by peer review) is the author/funder, who has granted bioRxiv a license to display the preprint in perpetuity. It is made available under aCC-BY-NC 4.0 International license.

a

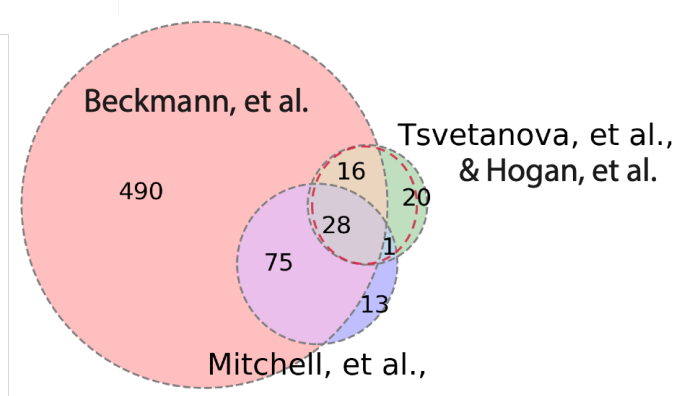

b

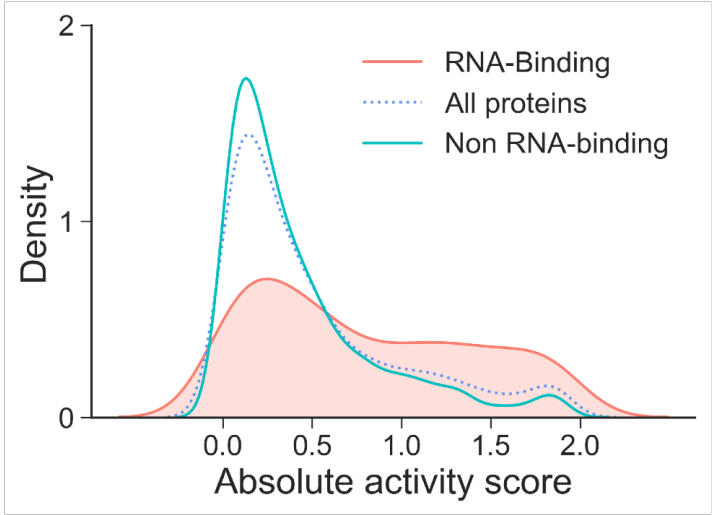

d

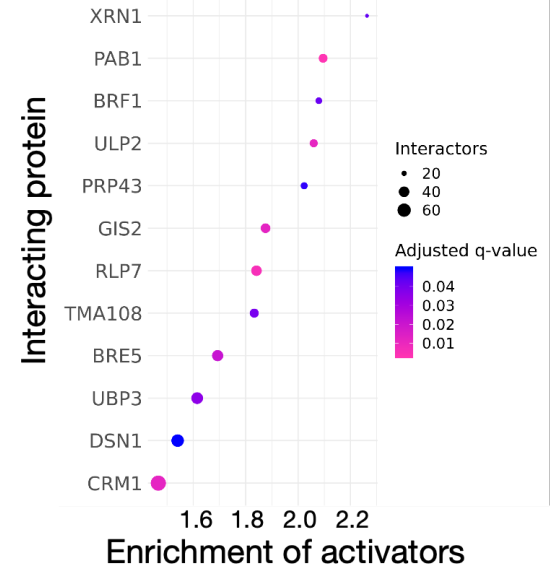

c
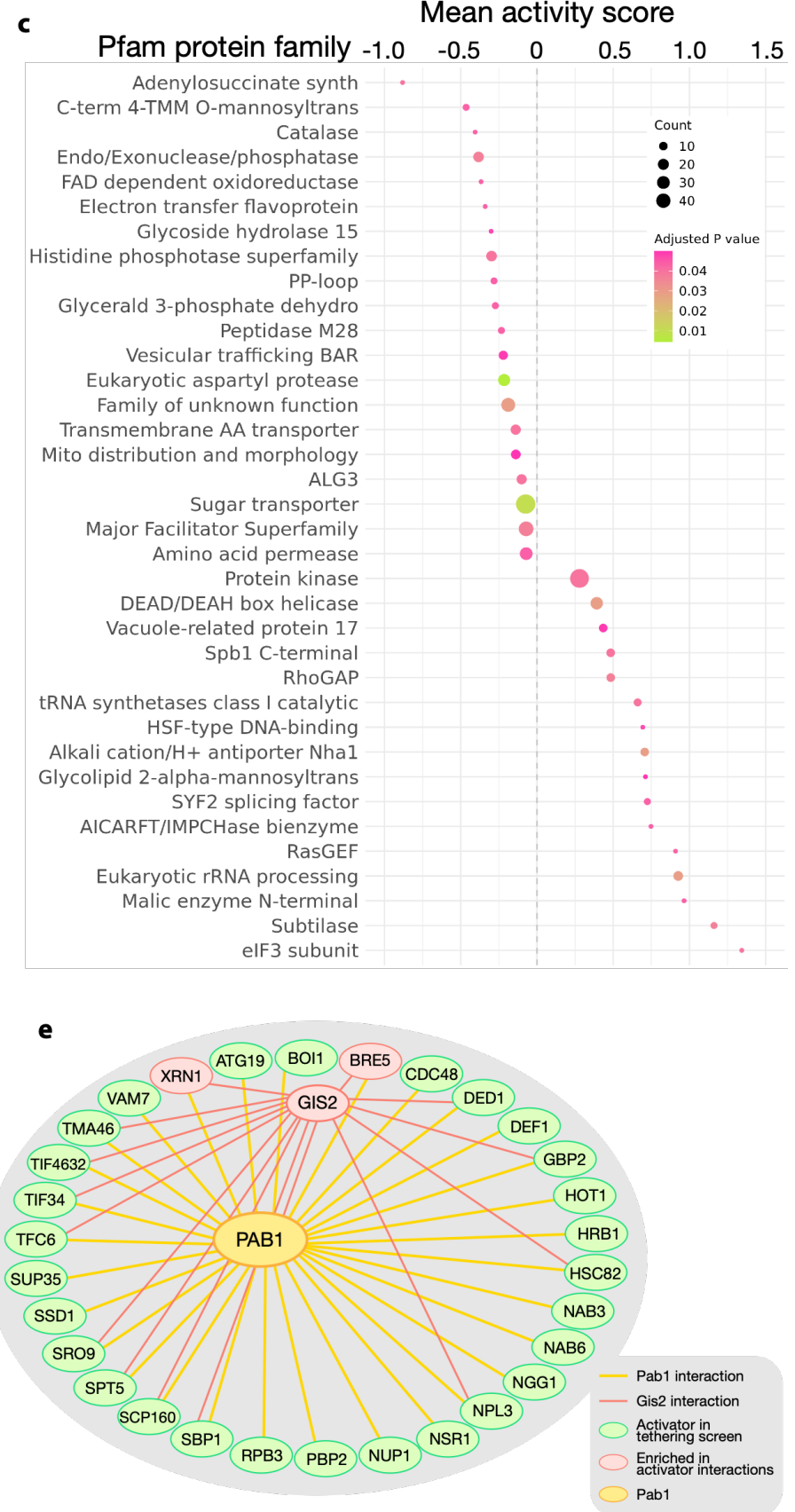

Fig. 4: Global analyses reveal enrichment of protein domains, motifs and protein-protein interactions amongst most active screen fragments.

a, Venn diagram of overlapping datasets identifying RBPs. b, KDE of absolute activity scores for RNA-binding proteins, all proteins, and non-RNA-binding proteins in the screen. c, Pfam protein domains significantly enriched amongst active screen fragments. d, Plot of proteins significantly enriched in interactions with activator fragments, $\mathrm{x}$-axis represents fold-enrichment. e, Pab1 interactions with activator fragments (outer ring) with overlapping Gis2 interactions depicted in red.

important roles in post-transcriptional

regulation, and the presence of IDRs in an RBP is often an evolutionarily conserved feature of the protein ${ }^{47}$. In some cases, IDRs participate in forming protein-protein interactions and can adopt a more stable conformation upon ligand binding, as in the case of the disordered Nterminus of Ded1 48,49 , as well as serving as flexible linkers 50,51 . Functional IDRs can include short linear interaction motifs (SLiMs), which are 
often responsible for protein-protein interactions 52. Though SLiMs cannot be recognized in bioinformatic searches for protein domains, they may be recognizable as peptide sequence motifs.

Motivated by the possibility that SLiMs could explain regulatory effects, we searched for peptide motifs enriched in our highly-active fragments. We carried out this search using MEME, and then scanned the yeast proteome for occurrences of these motifs using FIMO ${ }^{53}$. In some cases, these motifs were associated with highly repetitive elements. While these occurrences may reflect true regulatory activity, their interpretation is difficult because they are repetitive, so they were excluded. We identified six motifs that were statistically enriched amongst our activators and repressors (Extended Data Fig. 4a, b and Extended Datasets 7-8). These motifs align to genes with functions spanning many aspects of cell biology, including cell wall maintenance, cytoskeleton functions, transcription, and translation. The glutaminerich motif (repressor motif 2 in Extended Data Fig. 4a) is particularly enriched in genes involved in mRNA metabolism, for example NGR1, POP2, and PUF3, which all have diverse roles in mRNA deadenylation and decay 31,54,55. Likewise, the RGG repeat in activator motif 5 (Extended Data Fig. 4b) is widespread among RBPs and linked to post-transcriptional regulation ${ }^{56}$.

Regulatory RBPs often exert their effects by recruiting and activating core cellular machinery involved in translation and RNA decay. It seemed plausible that many of the active regulators we uncovered functioned in this way as well by forming protein-protein interactions with endogenous partners. We thus expected that several different active fragments from our screen might share common interactors. We intersected our library fragments with the collection of physical protein-protein interactions in the BioGRID database 57 and searched for proteins with a significant over-representation of activating or repressing fragments amongst their interactors. We identified a dozen such proteins (Extended Dataset 9), all enriched for interaction with activators, and most with clear ties to RNA biology (Fig. 4d). Strikingly, the poly-(A) binding protein Pab1 showed one of the highest degrees of enrichment, consistent with its central role in mRNA translation activation 19,58. The translation regulator Gis2 59,60 was also substantially enriched in activators, and shared many interaction partners with Pab1 (Fig. 4e). Somewhat surprisingly, the exonuclease Xrn1 exhibited the strongest enrichment in interactions with activators (Fig. 4d) despite its role in mRNA decay ${ }^{61}$. This enrichment may reflect a common core of mRNA-binding proteins that accompany transcripts during both translation and degradation. However, Xrn1 does promote the translation of a specific subset of transcripts encoding membrane proteins, and so this enrichment might also represent a more direct effect 62 .

\section{$E R / G o l g i$ protein Gtal is a bimodal post- transcriptional repressor}

Several overlapping, C-terminal fragments of the protein Gta1 emerged as potent repressors detected in our tethering screen (Extended Dataset 5). This region of Gta1 also harbored one of the repressor-associated peptide motifs that we identified in our library (Fig. $4 b$ and $5 a$ ). While the Gta1 protein co-purifies with the translational machinery ${ }^{33}$, genetic evidence suggests that GTA1 plays a role in golgi and vesicle transport 63,64, and the Gta1-GFP fusion protein localizes to the ER $65-67$. Due to its reported association with ribosomes and the presence of a repressive motif, we chose to further investigate Gta1. We generated $\lambda \mathrm{N}$ fusion constructs of both Gta1(603-767), the strong repressive fragment in our library, and full-length Gta1 protein, and tested their effects on reporter expression (Fig. 5b).

Both full-length Gta1 and the Gta1(603-767) fragment robustly reduced median YFP and produced a strongly bimodal distribution of reporter expression (Fig. 5c), a distinctive pattern 

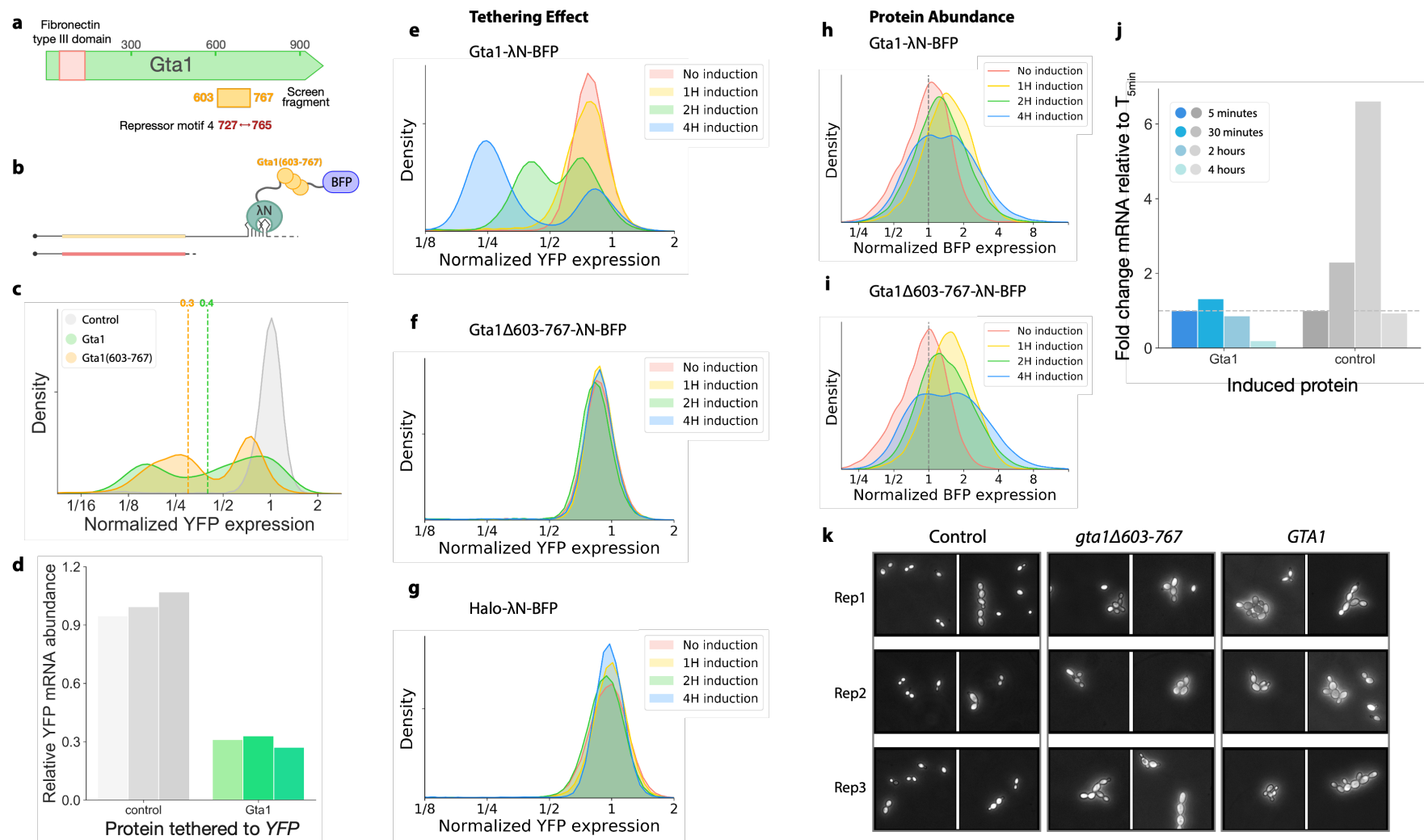

Fig. 5: The tethering screen identifies RNA-regulatory roles of poorly-characterized proteins.

a, Schematic representation of Gta1 protein with the C-terminal Gta1(603-767) fragment highlighted. $\mathbf{b}$, Schematic depiction of Gta1(603-767) in the tethering assay. c, Flow cytometry measuring activity of Gta1 and Gta1(603-767) in the tethering assay, dotted lines represent median YFP expression $(n=2$, one replicate per sample shown). $d$, RT-qPCR analysis of YFP mRNA abundance with Gta1 tethered to the $3^{\prime}$ UTR, normalized to a non-regulator control (n=3). e-g, Time course of reporter changes after induction of $\mathbf{e}$, Gta1 $\mathbf{f}$, Gta1 $\triangle 603-767$ and $\mathbf{g}$, Halo control tethering constructs $(\mathrm{n}=2)$. $\mathbf{h}$, Change in BFP fluorescence as a measure of Gta1 expression over time, normalized to the uninduced Gta1 sample ( $\mathrm{n}=2$, one replicate shown per time point). $\mathbf{i}$, As in $\mathbf{h}$, for Gta1 $\triangle 603-767$. j, RT-qPCR analysis of induced GTA1 and control mRNA over time, normalized to expression levels at 5 minutes induction. k, Light microscopy of yeast overexpressing GTA1, GTA1 $6603-767$, or Halo control for 4 hours.

that we did not see for any other tethering construct we examined individually. As expression of the isolated Gta1(603-767) fragment appeared to slow cell growth, we focused our analysis on full-length Gta1. We found that Gta1 tethering greatly reduced reporter mRNA abundance (Fig. 5d), suggesting that its regulatory effect principally reflected increased mRNA turnover. We next wished to track how bimodality emerged when the Gta1$\lambda \mathrm{N}$ fusion was switched on acutely, and so we expressed it from an inducible promoter. Levels of the YFP reporter began to decline within one hour of inducing the tethering construct, and clear bimodality emerged within two hours (Fig. 5e). Reporter levels in the lower peak continued to decline over the next two hours, likely reflecting the loss of pre-existing YFP through degradation or dilution. Notably, deletion of the repressor fragment that we identified in a Gta1 $\Delta 603-767-\lambda N$ tethering construct abolishes this effect entirely (Fig. $5 \mathrm{f}$ and $5 \mathrm{~g}$ ). We thus confirm that the Gta1(603-767) region containing our repressive peptide motif is both necessary and sufficient for the regulatory effect.

We next tested whether the bimodal reporter expression we observed was a result of variation in the abundance of the Gta1 tethering fusion in the population. Indeed, we saw a broad, bimodal distribution of BFP fluorescence from the Gta1$\mathrm{BFP}-\lambda \mathrm{N}$ construct after four hours of induction 
(Fig. 5h). Furthermore, it appeared that Gta1$\mathrm{BFP}-\lambda \mathrm{N}$ levels increased more uniformly in the first hour of induction, followed by the emergence of two distinct phenotypes in this genetically homogeneous population (Fig. 5h). Notably, we saw a very similar trajectory after induction of the Gta1 $\Delta 603-767-\lambda N$ tethering construct (Fig. 5i), although this tethering construct did not affect YFP expression (Fig. 5f). We also measured mRNA abundance of inducible GTA1, which quickly rose to 20 times the endogenous levels of GTA1 in an uninduced control (Extended Data Fig. 5b). Levels of the inducible mRNA did not further increase, and actually declined substantially after 4 hours in the continuous presence of the inducer (Fig. 5j). In contrast, levels of the mRNA encoding the inactive Halo-BFP- $\lambda N$ tethering construct used as a control in our experiments increased substantially in the two hours following induction (Fig. 5j). These mRNA abundance measurements reflect population averages, whereas flow cytometry highlights the cell-tocell variability underlying these responses.

The heterogeneity we saw by flow cytometry led us to observe the effects of GTA1 overexpression by light microscopy. We noted that induction of full-length GTA1, or the GTA1 $\triangle 603-767$ mutant lacking RNA destabilization activity, cause an atypical, elongated morphology and persistent chains and clusters of cells, akin to the filamentous or pseudohyphal growth that $S$. cerevisiae can undergo upon nutrient limitation (Fig. 5k) 68,69 . Since Gta1 $\Delta 603-767$ is not a strong post-transcriptional repressor, however like fulllength Gta1 it appears to impact budding morphology and declines in protein abundance during prolonged transcriptional induction (Fig. 5i), the role for Gta1 in RNA turnover appears separable from its impact on budding.

\section{Intrinsically disordered regions mediate the activity of the translation initiation factor Ded1 and the deadenylase Ccr4}

By identifying active fragments within larger

proteins, we were able to distinguish the features and domains with the strongest regulatory effects. For example, our library contained many fragments of the exonuclease Ccr4, one of two proteins responsible for the deadenylase activity of the Ccr4-Not complex and thus a key factor in eukaryotic mRNA turnover ${ }^{70}$. Consistent with the role of Ccr4 in deadenylation and thus mRNA decay, we identified many repressive Ccr4-derived fragments. Indeed, the median activity score of all of the assayed Ccr4 fragments was -0.5 (Fig. 6a), and the strongest repressive fragment in the screen, Ccr4(2-203), had an activity score of -1.8 (Fig. $6 \mathrm{~b}$ ). This strongly repressive fragment derived from the $\mathrm{N}$-terminus of Ccr4, however, rather than the Cterminal nuclease domain 71,72 . We obtained excellent coverage of the N-terminal half of Ccr4 (Fig. 6c), allowing us to observe that the disordered N-terminus itself, including the first 200 amino acids of the protein, yielded the strongest repressors, while the downstream folded leucine-rich repeat had little activity on its own (Fig. 6c). Our results suggest a regulatory role for the disordered N-terminus, which is not required for the nuclease activity of $\mathrm{Ccr} 4$ or its assembly into the Ccr4-Not complex ${ }^{72}$.

A similar pattern emerged among the regulatory fragments derived from the translation initiation factor Ded1. This protein is a highly-conserved RNA helicase of the DEAD-box family that interacts with core translation initiation factors in the cap-binding eIF4F complex and is important for translation of many yeast mRNAs $48,73,74,49$. Ded1 fragments appeared amongst the strongest of the post-transcriptional activators in the tethering screen, consistent with its positive role in mRNA expression (Fig. 6d). We obtained excellent resolution across the disordered $\mathrm{N}$ terminus of Ded1 and observed a consistent increase in activity for longer fragments (Fig. 6e and F). These results are consistent with deletion analyses that identified two distinct regions of the N-terminus required for interactions with translation initiation factors eIF4A and eIF4E 48. Our results indicate that full activity of Ded1 
a

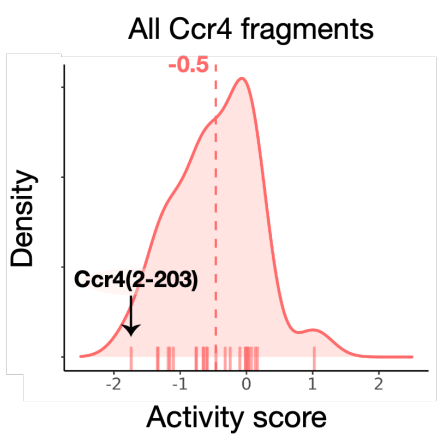

b
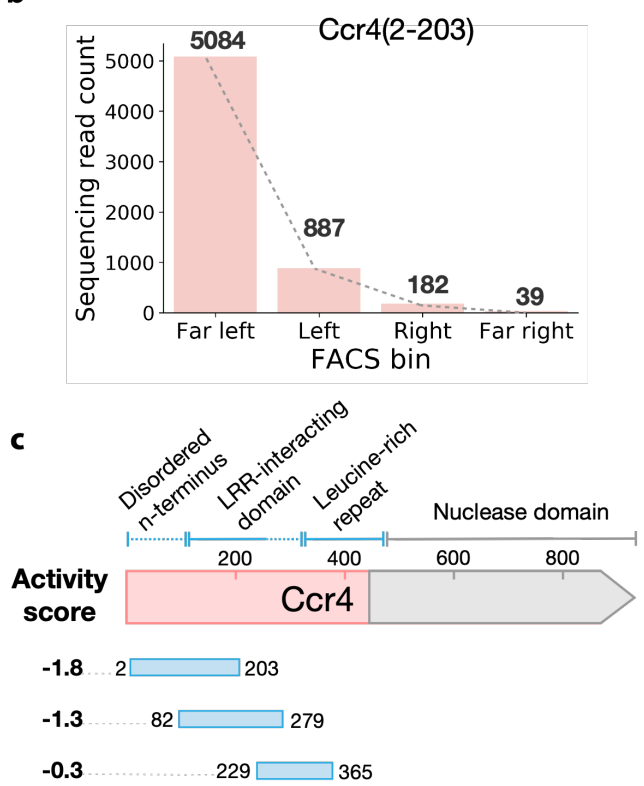

fragments in the tethered-function assay requires both of these interactions, mediated by Ded1(30-60) and Ded1(60-100), respectively. Our analysis of Ded1 and Ccr4 demonstrates the power of our approach in delineating active domains and peptide motifs within proteins and emphasizes that strong regulatory effects are often associated with disordered interaction motifs.

\section{The tethering screen identifies functions of the RBPs Sro9 and Cdc48}

We identified and validated strong, positive post-transcriptional regulatory activity in an $\mathrm{N}$ terminal fragment of the RNA-binding protein Sro9 (Fig. 3b, e). This poorly characterized protein, one of three La-motif-containing proteins in yeast, associates with translating ribosomes 75 and translation initiation factors 35,76 . It appears to bind and stabilize target mRNAs enriched for functions in protein synthesis ${ }^{35}$. We noted that Sro9 also contains one of the activator-associated peptide motifs we identified (Extended Data Fig. 4b, Fig. 7a), although our validated N-terminal fragment did not include this motif. In order to further dissect the regulatory activity of Sro9, we tested the fulllength protein along with one truncation, Sro9(1-151), that encompassed our validated fragment, and a longer Sro9(1-251) truncation that included the activator-rich motif as well. We also tested the remaining C-terminal half of the protein, Sro9(252-434), which includes the Lamotif and is implicated in RNA binding ${ }^{35}$ (Fig. 7Aa). We tethered each construct to the $3^{\prime}$ UTR of our reporter using a $\lambda \mathrm{N}$ fusion and examined their impact on its expression. Interestingly, inclusion of the activator-associated motif did not further increase the activity of the Sro9 Nterminus, although full-length Sro9 was a substantially stronger activator (Fig. 7b). The C- 
bioRxiv preprint doi: https://doi.org/10.1101/2021.08.09.455688; this version posted August 9, 2021. The copyright holder for this preprint (which was not certified by peer review) is the author/funder, who has granted bioRxiv a license to display the preprint in perpetuity. It is made available under aCC-BY-NC 4.0 International license.

Reynaud et al.

a

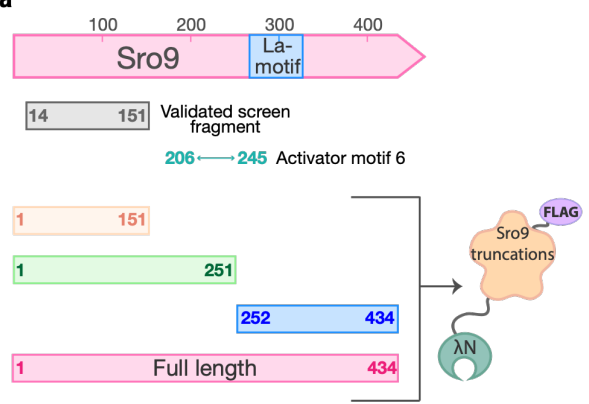

b

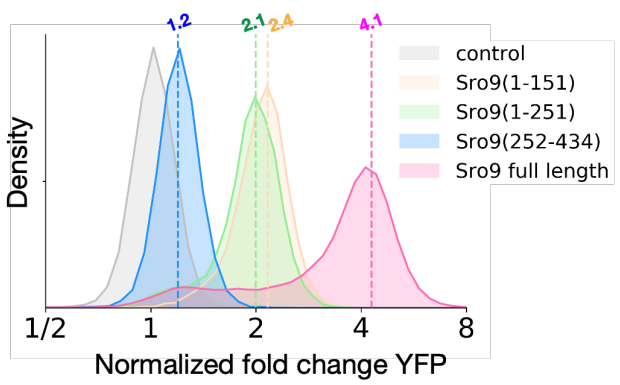

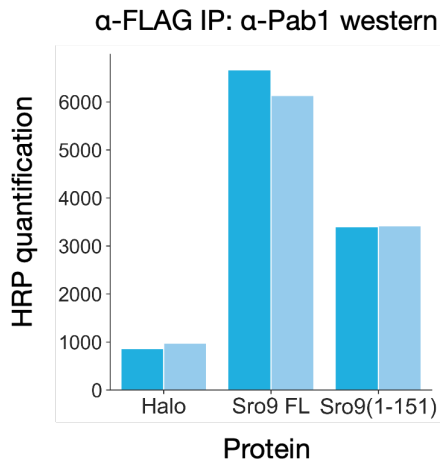

f

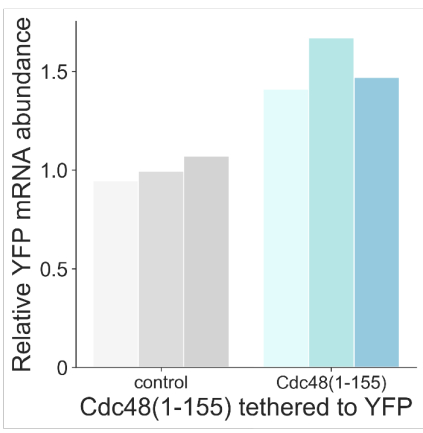

j

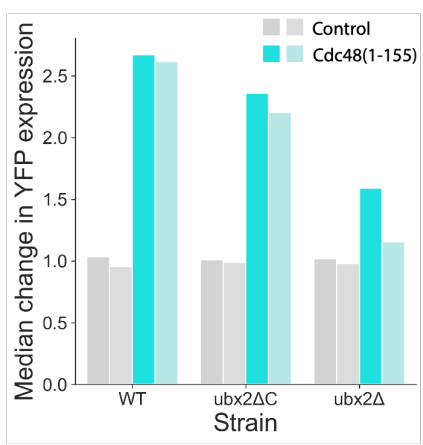

g
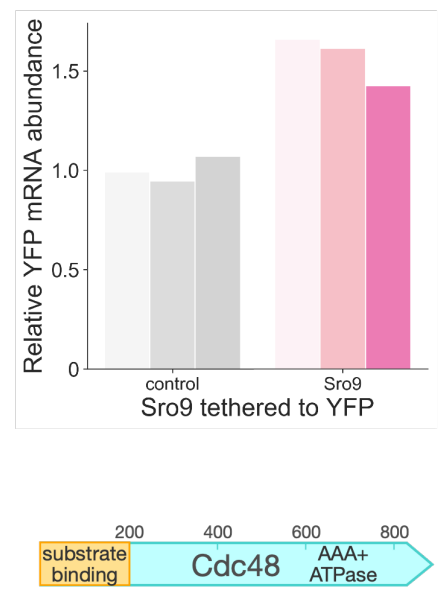

$\begin{array}{ll}1 & 155\end{array}$ Screen fragment

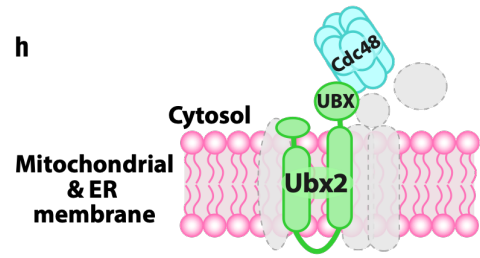

e

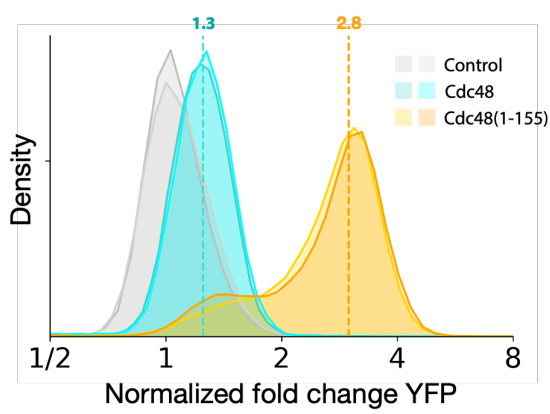

i

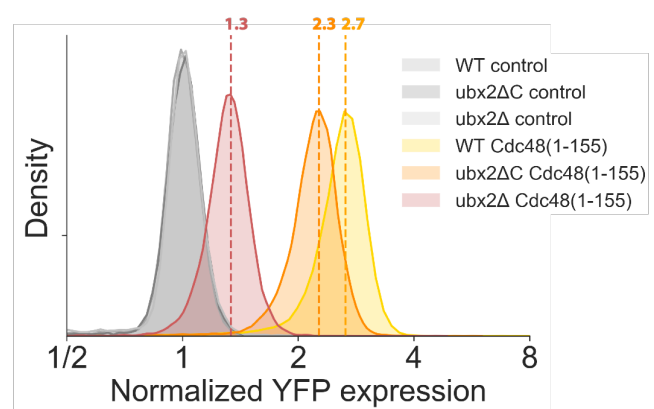

Fig. 7: The tethering screen reveals regulatory roles of known RNA-binding proteins.

a, Schematic representation of Sro9 protein domains and its truncations characterized in the tethering assay. $\mathbf{b}$, Flow cytometry measuring activity of Sro9 full length and truncations in the tethering assay, dotted lines represent median YFP expression ( $n=2$, one replicate per sample shown). $c$, Quantification of horseradish peroxidase (HRP) western blot analysis for Pab1 enrichment in FLAG-tag immunoprecipitation eluate $(n=2)$. $d$, RT-qPCR analysis of YFP mRNA abundance with Sro9 tethered to the 3' UTR, normalized to a non-regulator control ( $n=3)$. e, As in $\mathbf{b}$, for Cdc48 and Cdc48(1-155) ( $n=2)$. $\mathbf{f}$, As in $\mathbf{d}$, for Cdc48(1-155). g, Schematic representation of Cdc48 protein with its substrate, cofactor and ubiquitin binding Nterminal domain highlighted. $\mathbf{h}$, Schematic depiction of Cdc48 recruitment to the ER and mitochondrial membranes through Ubx2. i, As in b, for Cdc48(1-155) in the wild-type, $u b x 2 \Delta C$, and $u b x 2 \Delta$ strains ( $\mathrm{n}=2$, one replicate per sample shown). $\mathbf{j}$, Quantification of median values in $\mathbf{i}$.

terminal portion alone was essentially inactive, which is consistent with our hypothesis that the RNA-binding regions of RBPs function more in cargo selection than eliciting regulatory effects. All of the fusion constructs were robustly expressed (Extended Data Fig. 7a) such that the stronger effect of the full-length protein cannot be explained by protein stability. Instead, these results point to some other way that the context of full-length Sro9 potentiates the positive effects of the N-terminal region.

Sro9 is reported to interact with several translation factors, including Pab1, which emerged as a common interaction partner for 
many activators in the screen (Fig. $4 \mathrm{~d}$, e) ${ }^{35}$. We thus wanted to test whether the Sro9(1-151) fragment was sufficient for a stable Pab1 interaction. Indeed, we found that Pab1 copurified with epitope-tagged N-terminal Sro9(1-151) (Fig. 7c and Extended Data 7b). The co-purification of Pab1 with full-length Sro9 protein was somewhat stronger, consistent with the stronger activation seen from tethered fulllength Sro9 protein than from the N-terminal region alone. As Pab1 can enhance expression by stabilizing mRNAs or by promoting their translation, we wanted to test how Sro9 tethering affected mRNA abundance. We found that Sro9 increased YFP mRNA abundance by only $~ 1.5-$ fold (Fig. 7d), indicating that increased translation explains the majority of its regulatory effect. These results are consistent with the modest quantitative changes in transcript abundance observed in sro9 $\Delta$ yeast 35 .

While the translation activation we observed from the Sro9 N-terminus was consistent with its known function, we also observed positive effects upon tethering proteins with little known role in mRNA regulation. Notably, we found that an N-terminal fragment of the AAA ATPase Cdc48 increased reporter expression. Cdc48 has many roles in the cell, but is linked most prominently with protein degradation, including the ER associated degradation (ERAD) pathway for quality control of transmembrane and secreted proteins ${ }^{77}$. Cdc48 acts as an unfoldase that extracts proteins from membranes and complexes in order to make them available for proteasomal degradation ${ }^{78-81}$. Despite these strong connections with protein turnover, Cdc48 was recently reported to interact with RNA 9, although its role in RNA regulation, and the identities of the specific transcripts that it binds, remain mysterious. Known functions of Cdc48 also seem to suggest that it would negatively regulate protein expression.

We were thus surprised to discover that tethering the N-terminal Cdc48(1-155) fragment to a reporter transcript robustly activated its expression (Fig. 7e and Extended Dataset 5). Furthermore, this appeared to result from enhanced translation, as reporter mRNA levels were only modestly increased by Cdc48(1-155) tethering (Fig. 7f). Interestingly, full length Cdc48 did not show this same activity (Fig. 7e). The Nterminus of Cdc48 spanning approximately the first 200 amino acids binds to substrates, cofactors, and ubiquitin, while the C-terminal domains form the hexameric AAA ATPase 77 (Fig. 7g). Our results thus suggest that cofactor interactions of the isolated N-terminus could increase translation. One such cofactor is the UBX-domain-containing protein Ubx2, which localizes Cdc48 to the sites of ERAD and mitochondrial protein translocation-associated degradation (mitoTAD) ${ }^{82-84}$ (Fig. 7h). Deletion of the UBX2 gene $(u b \times 2 \Delta)$ reduced $C d c 48(1-155)$ activity dramatically (Fig. 7i, j). Removal of the C-terminal UBX-domain, which mediates the interaction between $\mathrm{Ubx} 2$ and Cdc48 83 , had a smaller impact on Cdc48(1-155) activity in the tethering assay. It is possible that the localization of Cdc48(1-155) to the ER or mitochondrial surface may recruit the tethered reporter transcript and thereby modulate its expression. Alternately, the isolated N-terminus could displace binding of full-length Cdc48 from other recruitment factors on the mRNA.

\section{DISCUSSION}

Here we report a broad and unbiased survey of the budding yeast proteome that identifies proteins controlling mRNA translation and decay. We recover a wide array of known and novel regulators, strongly enriched for RNAbinding proteins, that illustrates the breadth of post-transcriptional gene regulation. We also delineated the active regions within these proteins at domain-level resolution, which revealed that regulatory activity typically maps outside the RNA-binding domains themselves. Post-transcriptional regulators thus seem to display a modular architecture, with RNAbinding domains that determine their mRNA 
specificity and separate regulatory domains that modulate the expression of these target transcripts 5,85 . We find regulatory activity associated with folded domains, but also with disordered regions, highlighting the importance of functional IDRs in post-transcriptional regulation ${ }^{85}$. Indeed, the most potent repressive fragments of the Ccr4 deadenylase included its disordered N-terminus, and disordered fragments from the $\mathrm{N}$-terminus of the translation initiation factor Ded1 strongly activated expression. Two broad models have emerged to explain how such IDRs might show specific molecular functions. General patterns of amino acid composition, such as interspersed acidic and aromatic residues, seem to underlie transcriptional activation by IDRs ${ }^{86-88}$. Other IDRs harbor short, linear motifs (SLiMs) that act through well-defined peptide-protein docking rather than more heterogeneous interactions based on polymer properties ${ }^{89}$. Both degenerate and specific peptide motifs emerged from our survey, suggesting that both modes of actions play important roles in mRNA regulation.

Many of the regulators we identified may exert their effect through their interactions with other proteins. This pattern held even in Ccr4 and Ded1, which both contain enzymatic activities that could act directly on a tethered mRNA. These interactions can affect expression of a target transcript by recruiting the large, multiprotein complexes involved in translation and mRNA decay or modulating their activity. Indeed, we observe that positive regulators were enriched for interaction with the poly-(A) binding protein Pab1, which stabilizes mRNAs and promotes their translation, suggesting that these regulators could converge onto core pathways controlling the fate of mRNAs. Similar patterns have been seen in organisms ranging from trypanosomes to humans, and so this convergence may reflect a general organizational principle of eukaryotic post-transcriptional regulation 16,17 .
The fluorescence-based tethering assay offers a tool to further explore these regulatory networks and more broadly understand the mechanistic basis for post-transcriptional regulation. Our data also provide the key to decipher the functional consequences of the diverse RNAbinding proteome that has recently come into view.

\section{MATERIALS AND METHODS}

\section{Strain construction}

The dual fluorescent reporter (YFP::PP7 / RFP::boxB) strain NIY289 was constructed as follows: pNTI252 was integrated into BY4741 at URA3 to generate NIY106. pNTI476 was integrated into BY4742 at URA3 to generate NIY287. NIY106 was crossed with NIY287 to create NIY293. The dual fluorescent reporter (YFP::boxB / RFP::PP7) strain NIY293 was constructed as follows: pNTI282 was integrated into BY4741 at URA3 to generate NIY114. pNTI473 was integrated into BY4742 at URA3 to generate NIY286. NIY114 was crossed with NIY286 to create NIY293. Wild-type BY4741 was used for the in-frame library selection. The yKS090 dual reporter strain expressing the ZIF synthetic transcription factor was generated by integrating pNTI727 into the yeast XII-5 integration site ${ }^{91}$. The UBX2 mutation strains were generated as follows: We amplified the KanMX cassette from pCfB2225 with primers to generate homologous overlapping sequences to the UBX2 locus in the yeast genome, and then integrated this cassette into one locus of UBX2 in NIY293. We verified correct chromosomal integration by colony PCR which indicated a heterozygous $u b x 2 \Delta / U B X 2$ genotype (yKS092). We then amplified the KILEU2 cassette from pCfB2189 with either homologous sequences to the remaining $U B X 2$ locus or to the $\mathrm{C}$-terminal UBX domain of $U B X 2$ and then integrated these amplicons into yKS092 to create the $u b x 2 \Delta$ and $u b \times 2 \Delta c$ strains, respectively (yKS093 and yKS094). Genotypes were confirmed through 
colony PCR. Plasmids and strains are listed in Supplementary Tables 1 and 2, respectively.

\section{Plasmids used in this study}

See Extended Data Table 1.

\section{Culturing conditions}

Cultures for the single protein tethering assay were grown to mid-exponential growth phase at $\mathrm{OD}_{600} 0.6$ then harvested via gentle centrifugation at $5000 \times \mathrm{g}$ for 1 minute for flow cytometry analysis with 30 minutes incubation in $4 \%$ paraformaldehyde (PFA). For in-frame fragment selection, cultures were incubated after transformation at $30^{\circ} \mathrm{C}$ with shaking for 96 hours in SCD -His media with twice-daily backdilution to avoid culture saturation. NIY293 was transformed with the in-frame tethered fragment library with the high-efficiency lithium acetate method ${ }^{93}$ and then transferred to a turbidostat 94 for 48 hours in SCD -His media before harvesting cells. Cultures for the inducible Gta1 tethering assay were grown to stationary phase overnight, back-diluted to $\mathrm{OD}_{600} 0.1 \mathrm{OD}$ and allowed to reach early exponential growth phase. The tethered proteins were then induced with $5 \mathrm{nM} \beta$-estradiol, then harvested and fixed in PFA as described above.

\section{Flow cytometric measurements and fluorescence-activated cell sorting}

Expression of YFP and RFP in the tethering assay were measured using flow cytometric readout on a BD LSR Fortessa X20 with excitation by the $488 \mathrm{~mm}$ blue laser and $561 \mathrm{~mm}$ yellow-green laser, captured on the FITC and PE-TexRed channels, respectively. Fluorescence measurements for 50,000 cells were collected per sample, and gates were drawn to include populations of the $\sim 25 \%$ cells with modal forward- and side-scatter. Fluorescence activated cell sorting was performed with an Aria Fusion sorter by gating four equal-sized populations based on the ratio of FITC and PE-TexRed emission. Approximately two million cells were sorted into each gate. The sort was performed with two technical replicate libraries from the same library transformation.

\section{In-frame and fragment tethering library generation}

Genomic yeast DNA was tagmented using the Nextera XT DNA library prep kit from Illumina, and then size-selected with Beckman AMPureXP beads. Size selection was confirmed with an Agilent Tapestation 2200 on a High Sensitivity D1000 Screentape (Fig. S2A). BY4741 yeast were then co-transformed with the tagmented yeast gDNA and linear pKS132 and cultured as described above. After a long outgrowth in selective media, plasmids were harvested with the Zymo yeast miniprep kit. The selected fragments were then amplified by PCR with primers KS605(GTAATTATCTACTTTTTACAACAAATcc tgcaggGGCTCGGAGATGTGTATAAGAGACAG ) and

KS630(CTGTCTCTTATACACATCTGACGCcGG AAGCGGAAGCGGAAGCCGCGCCGACGCAC AAAC) designed to anneal to the Nextera XT sites introduced during tagmentation and subcloned into the SbfI-linearized tethering library vector pKS137 by Gibson Assembly. This tethering library was propagated in DH10 $\beta$ cells. Barcodes were then introduced by Gibson assembly of N25 randomized oligonucleotide barcodes, amplified with

KS633(ACGAGGCGCGTGTAAGTTACAGGCA AGCGATCCGTCCGTAATACGACTCACTATAG CACG) and

KS634(GATCCTGTAGCCCTAGACTTGATAGC CATGACTTCAACTCAAGACGCACAGATATT ATAA) into the BamHI-linearized tethering library. Assembly reactions were transformed into DH10 $\beta$ and selected in liquid cultures at varying dilutions in order to obtain a transformant pool with roughly three barcodes per fragment. This library was transformed into NIY293 through the lithium acetate method as described in ${ }^{93}$. Transformations were used to inoculate a turbidostat and grown in selective SCD -His media for 48 hours before performing 
FACS on live cells. Library plasmid DNA was then harvested from sorted cells with the Zymo yeast plasmid prep kit, and then barcode RNA was in-vitro transcribed with the HiScribe ${ }^{\mathrm{TM}} \mathrm{T} 7$ High Yield RNA kit from New England Biolabs. RNA was harvested with the previously described phenol chloroform method ${ }^{95}$. All PCR reactions were performed using Q5 polymerase according to manufacturer protocols. Barcodes were amplified through a limited cycle PCR with Illumina dual-index primers. Barcodes were assigned to yeast fragment DNA with next generation sequencing using the PacBio Single Molecule Real-Time (SMRT) technology

(Extended Dataset 2).

Barcode quantification and sequencing analysis Sequencing data was processed using Cutadapt to remove sequencing adapter sequences. HISAT2 was used to align sequencing reads to the yeast genome to identify fragment DNA. Trimmed barcodes were then counted and tabulated as described in ${ }^{96}$. Barcodes that lacked at least 32 counts in one of the sorted gates were filtered out.

\section{RNA quantification}

Total RNA was harvested from triplicate cultures of each strain using the phenol chloroform method ${ }^{95}$. Quantification of $Y F P$ reporter RNA expression in the tethering assay was performed via RT-qPCR analysis by comparing YFP Ct values to RFP Ct values, and Ct differences for cells expressing an active tethering protein were compared to a tethered Halo protein control Ct differences. The fold change in GTA1 expression in the induced cultures (Fig. S7A) was compared to the endogenous GTA1 levels in the Haloexpressing control strain.

\section{Domain and Motif enrichment analysis}

The search for domain enrichment amongst the tethered library fragments proceeded as follows: active fragments were first considered as those with an activity score of less than -1 or more than +1 . Active fragments that were $90 \%$ or more similar to another fragment were considered overlapping, and the most highly sequenced from a group of overlapping fragments was used in the analysis. A given Pfam protein domain was considered represented if one or more fragments covered at least $75 \%$ of the domain. The activity scores of represented protein domains were averaged and the mean value was reported for each domain. False discovery rates (FDRs) were calculated with the BenjaminiHochberg Procedure, and the domains with an adjusted p-value of less than 0.05 were reported in Fig. 4C.

To search for short peptide motifs enriched in our active fragments, we again considered active fragments as those with an activity score of less than -1 or greater than +1 . We then ran MEME analysis to search for recurring motifs within the sequences of our active fragments 53 . We collapsed sequences that were $50 \%$ or more similar into the same fragment in order to avoid detecting a motif multiple times within the same gene. We then used FIMO 53 to scan the yeast genome for occurrences of the motifs that were enriched in our active fragments. We manually removed two motifs which came from a single peptide sequence since these did not represent a consensus sequence from multiple distinct proteins, and we removed alignments that fell within highly-repetitive genomic sequences.

\section{Protein expression analysis via Western blotting}

Total protein was isolated from midexponentially growing yeast by rapid capture of protein expression through 5\% tricarboxylic acid treatment for ten minutes, followed by a wash in acetonitrile. The cell pellets were then dried at room temperature for 30 minutes before beadbeating in Tris-acetate-EDTA buffer for 5 minutes at room temperature. Samples were then resuspended in SDS-loading buffer from NuPage, boiled for five minutes, and loaded on 4-12\% polyacrylamide Bis-Tris gels and separated by electrophoresis in MOPS buffer. Proteins were then transferred to a nitrocellulose membrane and were blocked for 1 hour in TBST 
with 5\% bovine serum albumin. Primary antibodies were incubated with membranes at a 1-to-1000 dilution in TBST for one hour at roomtemperature, washed with TBST, and then incubated for 30 minutes at room temperature with anti-rabbit and anti-mouse HRP-linked antibodies at a 1-to-10000 dilution. Membranes were developed with Pierce ECL western blotting substrate and imaged on the chemiluminescence channel on a ProteinSimple.

\section{Microscopy}

Mid-exponential phase cells were harvested by gentle centrifugation and then fixed for 30 minutes in $4 \%$ PFA. The cells were washed in $1 x$ PBS buffer and visualized with a Leica DM IL LED microscope at 40X magnification. Fields of view for saved images were randomly selected.

\section{ACKNOWLEDGEMENTS}

We thank Shintaro Iwasaki and Gloria Brar for insightful comments and suggestions. We thank Ryan Muller for library plasmids, Samantha Fernandez for assistance with computational data visualization, and Joe Lobel along with other members of the Ingolia lab for thoughtful scientific discussion. This work was supported by NIH grants DP2 CA195768 (N.T.I.) and R01 GM130996 (N.T.I.) and by the Rose Hills Innovator Program. This work used the Vincent J. Coates Genomics Sequencing Laboratory at UC Berkeley, supported by NIH S10 OD018174 Instrumentation Grant, as well as the Flow Cytometry Facility at UC Berkeley, the UC Berkeley DNA Sequencing Facility, and the UC Davis Proteomics Core Facility.

\section{AUTHOR CONTRIBUTIONS}

\author{
Conceptualization: KR, AM, NI \\ Data curation: KR, NI \\ Formal analysis: KR, DN, NI \\ Funding acquisition: NI \\ Investigation: $\mathrm{KR}, \mathrm{AM}, \mathrm{ZM}$ \\ Methodology: KR, AM, NI \\ Project administration: KR, NI \\ Resources: NI
}

Supervision: NI

Validation: KR

Visualization: KR

Writing - original draft: KR, NI

Writing - review \& editing: KR, AM, NI

\section{DECLARATION OF INTERESTS}

The authors declare no competing interests.

\section{DATA AVAILABILITY}

- High-throughput sequencing data has been deposited with the NCBI Short Read Archive under BioProject PRJNA579846.

- Custom software and scripts are available from Zenodo at https: / / doi.org/10.5281/ zenodo. $\underline{4963329}$

- Plasmids will be made available through Addgene.

- Yeast strains described in this study are available upon request from the corresponding author.

\section{REFERENCES}

1. Gebauer, F., Preiss, T. \& Hentze, M. W. From cisregulatory elements to complex RNPs and back. Cold Spring Harb. Perspect. Biol. 4, a012245 (2012).

2. Hafner, M. et al. Transcriptome-wide identification of RNA-binding protein and microRNA target sites by PAR-CLIP. Cell 141, 129-141 (2010).

3. Gerstberger, S., Hafner, M. \& Tuschl, T. A census of human RNA-binding proteins. Nat. Rev. Genet. 15, 829-845 (2014).

4. Ott, M. Cell biology: Choreography of protein synthesis. Nature vol. 533 472-473 (2016).

5. Lunde, B. M., Moore, C. \& Varani, G. RNA-binding proteins: modular design for efficient function. Nat. Rev. Mol. Cell Biol. 8, 479-490 (2007).

6. Kramer, K. et al. Photo-cross-linking and highresolution mass spectrometry for assignment of RNAbinding sites in RNA-binding proteins. Nat. Methods 11, 1064-1070 (2014).

7. Lambert, N. et al. RNA Bind-n-Seq: quantitative assessment of the sequence and structural binding specificity of RNA binding proteins. Mol. Cell 54, 887900 (2014).

8. Castello, A. et al. Insights into RNA biology from an atlas of mammalian mRNA-binding proteins. Cell 149, 1393-1406 (2012).

9. Beckmann, B. M. et al. The RNA-binding proteomes 
Reynaud et al.

from yeast to man harbour conserved enigmRBPs. Nat. Commun. 6, 10127 (2015).

10. Licatalosi, D. D. et al. HITS-CLIP yields genome-wide insights into brain alternative RNA processing. Nature 456, 464-469 (2008).

11. Van Nostrand, E. L. et al. A large-scale binding and functional map of human RNA-binding proteins. Nature 583, 711-719 (2020).

12. Hafner, M. et al. CLIP and complementary methods. Nature Reviews Methods Primers 1, 1-23 (2021).

13. Van Nostrand, E. L. et al. Principles of RNA processing from analysis of enhanced CLIP maps for 150 RNA binding proteins. Genome Biol. 21, 90 (2020).

14. Coller, J. M., Gray, N. K. \& Wickens, M. P. mRNA stabilization by poly $(\mathrm{A})$ binding protein is independent of poly $(\mathrm{A})$ and requires translation. Genes Dev. 12, 3226-3235 (1998).

15. Bos, T. J., Nussbacher, J. K., Aigner, S. \& Yeo, G. W. Tethered Function Assays as Tools to Elucidate the Molecular Roles of RNA-Binding Proteins. Adv. Exp. Med. Biol. 907, 61-88 (2016).

16. Luo, E.-C. et al. Large-scale tethered function assays identify factors that regulate mRNA stability and translation. Nat. Struct. Mol. Biol. 27, 989-1000 (2020).

17. Erben, E. D., Fadda, A., Lueong, S., Hoheisel, J. D. \& Clayton, C. A genome-wide tethering screen reveals novel potential post-transcriptional regulators in Trypanosoma brucei. PLoS Pathog. 10, e1004178 (2014).

18. Keryer-Bibens, C., Barreau, C. \& Osborne, H. B. Tethering of proteins to RNAs by bacteriophage proteins. Biol. Cell 100, 125-138 (2008).

19. Kessler, S. H. \& Sachs, A. B. RNA recognition motif 2 of yeast Pab1p is required for its functional interaction with eukaryotic translation initiation factor 4G. Mol. Cell. Biol. 18, 51-57 (1998).

20. Collart, M. A. The Ccr4-Not complex is a key regulator of eukaryotic gene expression. Wiley Interdiscip. Rev. RNA 7, 438-454 (2016).

21. Finoux, A.-L. \& Séraphin, B. In Vivo Targeting of the Yeast Pop2 Deadenylase Subunit to Reporter Transcripts Induces Their Rapid Degradation and Generates New Decay Intermediates*. J. Biol. Chem. 281, 25940-25947 (2006).

22. Winstall, E., Sadowski, M., Kuhn, U., Wahle, E. \& Sachs, A. B. The Saccharomyces cerevisiae RNAbinding protein Rbp29 functions in cytoplasmic mRNA metabolism. J. Biol. Chem. 275, 21817-21826 (2000).

23. Adey, A. et al. Rapid, low-input, low-bias construction of shotgun fragment libraries by high-density in vitro transposition. Genome Biol. 11, R119 (2010).

24. Lu, B. et al. Transposase-assisted tagmentation of RNA/DNA hybrid duplexes. Elife 9, (2020).

25. Heger, A. \& Holm, L. Exhaustive enumeration of protein domain families. J. Mol. Biol. 328, 749-767 (2003).

26. Michlits, G. et al. CRISPR-UMI: single-cell lineage tracing of pooled CRISPR-Cas9 screens. Nat. Methods 14, 1191-1197 (2017).

27. Schmierer, B. et al. CRISPR/Cas9 screening using unique molecular identifiers. Mol. Syst. Biol. 13, 945 (2017).

28. Chuang, R. Y., Weaver, P. L., Liu, Z. \& Chang, T. H. Requirement of the DEAD-Box protein ded1p for messenger RNA translation. Science 275, 1468-1471 (1997).

29. De La Cruz, J., Iost, I., Kressler, D. \& Linder, P. The p20 and Ded1 proteins have antagonistic roles in eIF4E-dependent translation in Saccharomyces cerevisiae. Proceedings of the National Academy of Sciences 94, 5201-5206 (1997).

30. Tarn, W.-Y. \& Chang, T.-H. The current understanding of Ded1p/DDX3 homologs from yeast to human. RNA Biol. 6, 17-20 (2009).

31. Chang, L.-C. \& Lee, F.-J. S. The RNA helicase Dhh1p cooperates with Rbp1p to promote porin mRNA decay via its non-conserved C-terminal domain. Nucleic Acids Res. 40, 1331-1344 (2012).

32. Swaney, D. L. et al. Global analysis of phosphorylation and ubiquitylation cross-talk in protein degradation. Nat. Methods 10, 676-682 (2013).

33. Fleischer, T. C., Weaver, C. M., McAfee, K. J., Jennings, J. L. \& Link, A. J. Systematic identification and functional screens of uncharacterized proteins associated with eukaryotic ribosomal complexes. Genes Dev. 20, 1294-1307 (2006).

34. Brandariz-Núñez, A., Zeng, F., Lam, Q. N. \& Jin, H. Sbp1 modulates the translation of Pab1 mRNA in a poly(A)- and RGG-dependent manner. RNA 24, 43-55 (2018).

35. Kershaw, C. J. et al. The yeast La related protein Slf1p is a key activator of translation during the oxidative stress response. PLoS Genet. 11, e1004903 (2015).

36. Wendland, B. \& Emr, S. D. Pan1p, yeast eps15, functions as a multivalent adaptor that coordinates protein-protein interactions essential for endocytosis. J. Cell Biol. 141, 71-84 (1998).

37. Kaksonen, M. \& Roux, A. Mechanisms of clathrinmediated endocytosis. Nat. Rev. Mol. Cell Biol. 19, 313326 (2018).

38. Hogan, D. J., Riordan, D. P., Gerber, A. P., Herschlag, D. \& Brown, P. O. Diverse RNA-binding proteins interact with functionally related sets of RNAs, suggesting an extensive regulatory system. PLoS Biol. 6, e255 (2008).

39. Mitchell, S. F., Jain, S., She, M. \& Parker, R. Global analysis of yeast mRNPs. Nat. Struct. Mol. Biol. 20, 127-133 (2013).

40. Beckmann, B. M. RNA interactome capture in yeast. Methods 118-119, 82-92 (2017).

41. Tsvetanova, N. G., Klass, D. M., Salzman, J. \& Brown, P. O. Proteome-wide search reveals unexpected RNAbinding proteins in Saccharomyces cerevisiae. PLoS One 5, (2010).

42. El-Gebali, S. et al. The Pfam protein families database 
Reynaud et al.

in 2019. Nucleic Acids Res. 47, D427-D432 (2019).

43. Aitken, C. E. et al. Eukaryotic translation initiation factor 3 plays distinct roles at the mRNA entry and exit channels of the ribosomal preinitiation complex. Elife 5, (2016).

44. Linder, P. \& Jankowsky, E. From unwinding to clamping - the DEAD box RNA helicase family. Nature Reviews Molecular Cell Biology vol. 12 505-516 (2011).

45. Peng, Y. \& Weisman, L. S. The cyclin-dependent kinase Cdk1 directly regulates vacuole inheritance. Dev. Cell 15, 478-485 (2008).

46. Honzatko, R. B., Stayton, M. M. \& Fromm, H. J. Adenylosuccinate synthetase: recent developments. Adv. Enzymol. Relat. Areas Mol. Biol. 73, 57-102, ix-x (1999).

47. Varadi, M., Zsolyomi, F., Guharoy, M. \& Tompa, P. Functional Advantages of Conserved Intrinsic Disorder in RNA-Binding Proteins. PLoS One 10, e0139731 (2015).

48. Gulay, S., Gupta, N., Lorsch, J. R. \& Hinnebusch, A. G. Distinct interactions of eIF4A and eIF4E with RNA helicase Ded1 stimulate translation in vivo. Elife $\mathbf{9}$, (2020).

49. Gupta, N., Lorsch, J. R. \& Hinnebusch, A. G. Yeast Ded1 promotes $48 \mathrm{~S}$ translation pre-initiation complex assembly in an mRNA-specific and eIF4F-dependent manner. Elife 7, (2018).

50. Oberstrass, F. C. et al. Structure of PTB bound to RNA: specific binding and implications for splicing regulation. Science 309, 2054-2057 (2005).

51. Diarra dit Konté, N. et al. Aromatic side-chain conformational switch on the surface of the RNA Recognition Motif enables RNA discrimination. Nat. Commun. 8, 654 (2017).

52. Balcerak, A., Trebinska-Stryjewska, A., Konopinski, R., Wakula, M. \& Grzybowska, E. A. RNA-protein interactions: disorder, moonlighting and junk contribute to eukaryotic complexity. Open Biol. 9, 190096 (2019).

53. Bailey, T. L. et al. MEME SUITE: tools for motif discovery and searching. Nucleic Acids Res. 37, W2028 (2009).

54. Olivas, W. \& Parker, R. The Puf3 protein is a transcript-specific regulator of mRNA degradation in yeast. EMBO J. 19, 6602-6611 (2000).

55. Parker, R. \& Song, H. The enzymes and control of eukaryotic mRNA turnover. Nat. Struct. Mol. Biol. 11, 121-127 (2004).

56. Thandapani, P., O'Connor, T. R., Bailey, T. L. \& Richard, S. Defining the RGG/RG motif. Mol. Cell 50, 613-623 (2013).

57. Oughtred, R. et al. The BioGRID database: A comprehensive biomedical resource of curated protein, genetic, and chemical interactions. Protein Sci. 30, 187-200 (2021).

58. Sachs, A. The role of poly(A) in the translation and stability of mRNA. Current Opinion in Cell Biology vol.
2 1092-1098 (1990).

59. Rojas, M. et al. Yeast Gis2 and its human ortholog CNBP are novel components of stress-induced RNP granules. PLoS One 7, e52824 (2012).

60. Sammons, M. A., Samir, P. \& Link, A. J. Saccharomyces cerevisiae Gis2 interacts with the translation machinery and is orthogonal to myotonic dystrophy type 2 protein ZNF9. Biochem. Biophys. Res. Commun. 406, 13-19 (2011).

61. Parker, R. RNA degradation in Saccharomyces cerevisae. Genetics 191, 671-702 (2012).

62. Blasco-Moreno, B. et al. The exonuclease Xrn1 activates transcription and translation of mRNAs encoding membrane proteins. Nat. Commun. 10, 1298 (2019).

63. Costanzo, M. et al. A global genetic interaction network maps a wiring diagram of cellular function. Science 353, (2016).

64. Mattiazzi Usaj, M. et al. Systematic genetics and single-cell imaging reveal widespread morphological pleiotropy and cell-to-cell variability. Mol. Syst. Biol. 16, e9243 (2020).

65. Huh, W.-K. et al. Global analysis of protein localization in budding yeast. Nature 425, 686-691 (2003).

66. Chong, Y. T. et al. Yeast Proteome Dynamics from Single Cell Imaging and Automated Analysis. Cell 161, 1413-1424 (2015).

67. Kraus, O. Z. et al. Automated analysis of high-content microscopy data with deep learning. Mol. Syst. Biol. 13, 924 (2017).

68. Lorenz, M. C., Cutler, N. S. \& Heitman, J. Characterization of alcohol-induced filamentous growth in Saccharomyces cerevisiae. Mol. Biol. Cell 11, 183-199 (2000).

69. Cullen, P. J. \& Sprague, G. F., Jr. The regulation of filamentous growth in yeast. Genetics 190, 23-49 (2012).

70. Webster, M. W. et al. mRNA Deadenylation Is Coupled to Translation Rates by the Differential Activities of Ccr4-Not Nucleases. Mol. Cell 70, 10891100.e8 (2018).

71. Xu, K., Bai, Y., Zhang, A., Zhang, Q. \& Bartlam, M. G. Insights into the structure and architecture of the CCR4-NOT complex. Front. Genet. 5, 137 (2014).

72. Basquin, J. et al. Architecture of the nuclease module of the yeast Ccr4-not complex: the Not1-Caf1-Ccr4 interaction. Mol. Cell 48, 207-218 (2012).

73. Sen, N. D., Zhou, F., Ingolia, N. T. \& Hinnebusch, A. G. Genome-wide analysis of translational efficiency reveals distinct but overlapping functions of yeast DEAD-box RNA helicases Ded1 and eIF4A. Genome Res. 25, 1196-1205 (2015).

74. Guenther, U.-P. et al. The helicase Ded1p controls use of near-cognate translation initiation codons in 5' UTRs. Nature 559, 130-134 (2018).

75. Sobel, S. G. \& Wolin, S. L. Two yeast La motifcontaining proteins are RNA-binding proteins that 
associate with polyribosomes. Mol. Biol. Cell 10, 38493862 (1999).

76. Castelli, L. M. et al. The 4E-BP Caf20p Mediates Both eIF4E-Dependent and Independent Repression of Translation. PLoS Genet. 11, e1005233 (2015).

77. Baek, G. H. et al. Cdc48: a swiss army knife of cell biology. J. Amino Acids 2013, 183421 (2013).

78. Sommer, T. \& Wolf, D. H. Endoplasmic reticulum degradation: reverse protein flow of no return. FASEB J. 11, 1227-1233 (1997).

79. Kostova, Z. \& Wolf, D. H. For whom the bell tolls: protein quality control of the endoplasmic reticulum and the ubiquitin-proteasome connection. EMBO J. 22, 2309-2317 (2003).

80. Wolf, D. H. \& Stolz, A. The Cdc48 machine in endoplasmic reticulum associated protein degradation. Biochim. Biophys. Acta 1823, 117-124 (2012).

81. Olszewski, M. M., Williams, C., Dong, K. C. \& Martin, A. The Cdc48 unfoldase prepares well-folded protein substrates for degradation by the 265 proteasome. Commun Biol 2, 29 (2019).

82. Hartmann-Petersen, R. et al. The Ubx2 and Ubx3 cofactors direct $\mathrm{Cdc} 48$ activity to proteolytic and nonproteolytic ubiquitin-dependent processes. Curr. Biol. 14, 824-828 (2004).

83. Neuber, O., Jarosch, E., Volkwein, C., Walter, J. \& Sommer, T. Ubx2 links the Cdc48 complex to ERassociated protein degradation. Nat. Cell Biol. 7, 993998 (2005).

84. Mårtensson, C. U. et al. Mitochondrial protein translocation-associated degradation. Nature 569, 679683 (2019).

85. Gebauer, F., Schwarzl, T., Valcárcel, J. \& Hentze, M. W. RNA-binding proteins in human genetic disease. Nat. Rev. Genet. 22, 185-198 (2021).

86. Ravarani, C. N. et al. High-throughput discovery of functional disordered regions: investigation of transactivation domains. Mol. Syst. Biol. 14, e8190 (2018).

87. Staller, M. V. et al. A High-Throughput Mutational Scan of an Intrinsically Disordered Acidic Transcriptional Activation Domain. Cell Syst 6, 444455.e6 (2018).

88. Erijman, A. et al. A High-Throughput Screen for Transcription Activation Domains Reveals Their Sequence Features and Permits Prediction by Deep Learning. Mol. Cell 78, 890-902.e6 (2020).

89. Davey, N. E. et al. Attributes of short linear motifs. Mol. Biosyst. 8, 268-281 (2012).

90. Aranda-Díaz, A., Mace, K., Zuleta, I., Harrigan, P. \& El-Samad, H. Robust Synthetic Circuits for TwoDimensional Control of Gene Expression in Yeast. ACS Synth. Biol. 6, 545-554 (2017).

91. Stovicek, V., Borja, G. M., Forster, J. \& Borodina, I. EasyClone 2.0: expanded toolkit of integrative vectors for stable gene expression in industrial Saccharomyces cerevisiae strains. J. Ind. Microbiol.
Biotechnol. 42, 1519-1531 (2015).

92. Muller, R., Meacham, Z. A., Ferguson, L. \& Ingolia, N. T. CiBER-seq dissects genetic networks by quantitative CRISPRi profiling of expression phenotypes. Science 370, (2020).

93. Kawai, S., Hashimoto, W. \& Murata, K. Transformation of Saccharomyces cerevisiae and other fungi: methods and possible underlying mechanism. Bioeng. Bugs 1, 395-403 (2010).

94. McGeachy, A. M., Meacham, Z. A. \& Ingolia, N. T. An Accessible Continuous-Culture Turbidostat for Pooled Analysis of Complex Libraries. ACS Synth. Biol. 8, 844-856 (2019).

95. Nilsen, T. W. The fundamentals of RNA purification. Cold Spring Harb. Protoc. 2013, 618-624 (2013).

96. Muller, R., Meacham, Z. A., Ferguson, L. \& Ingolia, N. T. CiBER-seq dissects genetic networks by quantitative CRISPRi profiling of expression phenotypes. bioRxiv 2020.03.29.015057 (2020) doi: 10.1101/2020.03.29.015057. 